\title{
Dipole Collapse and Dynamo Waves in Global Direct Numerical Simulations
}

\author{
Martin Schrinner, Ludovic Petitdemange ${ }^{1}$ \\ and \\ Emmanuel Dormy \\ MAG(ENS/IPGP), LRA, École Normale Supérieure, 24 Rue Lhomond, 75252 Paris Cedex \\ 05, France \\ martin@schrinner.eu
}

\begin{abstract}
Magnetic fields of low-mass stars and planets are thought to originate from self-excited dynamo action in their convective interiors. Observations reveal a variety of field topologies ranging from large-scale, axial dipole to more structured magnetic fields. In this article, we investigate more than 70 three-dimensional, self-consistent dynamo models obtained by direct numerical simulations. The control parameters, the aspect ratio and the mechanical boundary conditions have been varied to build up this sample of models. Both, strongly dipolar and multipolar models have been obtained. We show that these dynamo regimes can in general be distinguished by the ratio of a typical convective length-scale to the Rossby radius. Models with a predominantly dipolar magnetic field were obtained, if the convective length scale is at least an order of magnitude larger than the Rossby radius. Moreover, we highlight the role of the strong shear associated with the geostrophic zonal flow for models with stress-free boundary conditions. In this case the above transition disappears and is replaced by a region of bistability for which dipolar and multipolar dynamos co-exist. We interpret our results in terms of dynamo eigenmodes using the so-called test field method. We can thus show that models in the dipolar regime are characterized by an isolated 'single mode'. Competing overtones become significant as the boundary to multipolar dynamos is approached. We discuss how these findings relate to previous models and to observations.
\end{abstract}

\footnotetext{
${ }^{1}$ Previously at Max-Planck-Institut für Astronomie, Königstuhl 17, 69117 Heidelberg, Germany
} 


\section{Introduction}

Topologies of stellar and planetary magnetic fields stemming from hydrodynamic dynamo action are highly variable. Observations revealed complicated field configurations dominated by higher multipoles, but also large-scale or even dipolar stellar and planetary magnetic fields are ubiquitous (Anderson et al. 2010; Hulot et al. 2010; Russell \& Dougherty 2010; Donati \& Landstreet 2009). The latter is the more astonishing, as the magnetic Reynolds number in planetary and stellar convection zones is often large and most hydrodynamic dynamos operate far above their threshold. Observations and numerical simulations indicate that rapid global rotation and thus the ordering influence of the Coriolis force is of major importance for the generation of coherent magnetic fields (Stellmach \& Hansen 2004; Käpylä et al. 2009; Brown et al. 2010). Kutzner \& Christensen (2002) demonstrated the existence of a dipolar and a multipolar dynamo regime and Christensen \& Aubert (2006) showed that the transition between both is governed by a local Rossby number, i.e. the influence of inertia relative to the Coriolis force. Similar results were reported by Sreenivasan \& Jones (2006), too. Dipolar models were found for small Rossby numbers; they are separated by a fairly sharp regime boundary from multipolar models, for which inertia is more important.

Sreenivasan \& Jones (2011) argued that dipolar magnetic fields enhance the kinetic helicity and are therefore easier to maintain than fields with a more complicated field topology. However, as noted by Sreenivasan \& Jones (2011), the relation between kinetic helicity and induction mechanisms is not straightforward. Moreover, Schrinner et al. (2007) showed that the kinetic helicity is indeed a bad proxy measure for the induction effects ( $\alpha$-effect) in these models.

Schrinner et al. (2010a) pointed out that the high relative dipole field strength for models in the low Rossby number regime is associated with the dominance of only one dipolar eigenmode. Spatially more complex modes were found to be strongly diffusive. In this dynamo regime, higher order contributions to the magnetic field result mainly from the deformation of the fundamental mode by the turbulent flow. Subsequently, the small scale contributions decay due to ohmic diffusion (Hoyng 2009; Schrinner et al. 2011). As a result of the dominance of only one, real eigenmode, the axis of the dipole field is stable, polarity reversals or oscillations of the magnetic field do not occur.

However, the reason for the dominance of an isolated eigenmode at low Rossby numbers and the cause of the dipole breakdown with decreasing influence of the Coriolis force are at present not well understood. In this study, we investigate how rotation influences the field topology in dynamo models in some more detail. We show that rotation-dominated convection in a spherical shell leads to a distinctive azimuthal field morphology which is well 
represented by the fundamental dynamo mode, but very different from the field pattern of the following higher-order modes. We argue that this discrepancy is responsible for the clear

preference of the fundamental mode over higher-order modes. Furthermore, we discuss how the dominance of the fundamental mode is reduced if convection becomes less affected by rotation and the typical length scale of convection decreases relative to the Rossby radius. Because the topology and the time variability of the magnetic field in numerical dynamo simulations are closely correlated we likewise address the question under which conditions models exhibit fairly coherent oscillations, irregular polarity reversals or a stable dipole field.

Our analysis is based on 72 dynamo models in the Boussinesq approximation with different aspect ratios and mechanical boundary conditions. We generalize here the Rossby number criterion given by Christensen \& Aubert (2006) to our sample of models. For some of them, we compute mean-field coefficients with the help of the test-field method (Schrinner et al. 2007) to reveal their dynamo mechanisms.

\section{Dynamo Calculations}

Our dynamo models are solutions of the MHD-equations for a conducting Boussinesq fluid in a rotating spherical shell. The fluid motion is driven by convection due to an imposed temperature difference, $\Delta T$, between the inner and the outer shell boundaries. The fundamental length scale of our models is the shell width $L$, we scale time by $L^{2} / \nu$, with $\nu$ the kinematic viscosity, and temperature is scaled by $\Delta T$. Moreover, following Christensen et al. (2001), the magnetic field is considered in units of $\sqrt{\varrho \mu \eta \Omega}$, with $\varrho$ denoting the density, $\mu$ the magnetic permeability, $\eta$ the magnetic diffusivity and $\Omega$ the rotation rate. With these units, the dimensionless momentum, temperature and induction equation are

$$
\begin{array}{r}
E\left(\frac{\partial \boldsymbol{v}}{\partial t}+\boldsymbol{v} \cdot \nabla \boldsymbol{v}-\nabla^{2} \boldsymbol{v}\right)+2 \boldsymbol{z} \times \boldsymbol{v}+\nabla P= \\
R a \frac{\boldsymbol{r}}{r_{o}} T+\frac{1}{P m}(\nabla \times \boldsymbol{B}) \times \boldsymbol{B}, \\
\frac{\partial T}{\partial t}+\boldsymbol{v} \cdot \nabla T=\frac{1}{P r} \nabla^{2} T, \\
\frac{\partial \boldsymbol{B}}{\partial t}=\nabla \times(\boldsymbol{v} \times \boldsymbol{B})+\frac{1}{P m} \nabla^{2} \boldsymbol{B} .
\end{array}
$$

We also note that $\boldsymbol{v}$ and $\boldsymbol{B}$ are solenoidal. The system of equations is governed by four dimensionless parameters, the Ekman number $E=\nu / \Omega L^{2}$, the (modified) Rayleigh number $R a=\alpha_{T} g_{0} \Delta T L / \nu \Omega$, the Prandtl number $\operatorname{Pr}=\nu / \kappa$, and the magnetic Prandtl number 
$P m=\nu / \eta$. In these definitions, $\alpha_{T}$ stands for the thermal expansion coefficient, $g_{o}$ is the gravitational acceleration at the outer boundary, and $\kappa$ is the thermal diffusivity. A further control parameter is the aspect ratio of the shell defined as the ratio of the inner to the outer shell radius, $\chi=r_{i} / r_{o}$. It determines the width of the convection zone.

The mechanical boundary conditions are either i) no slip at the inner and the outer boundary, ii) no slip at the inner and stress free at the outer boundary or iii) stress free at both boundaries. In the latter case, the angular momentum in the direction of the rotation axis was conserved. Furthermore, the magnetic field matches a potential field outside the fluid shell and fixed temperatures are prescribed at both boundaries.

Some of the models investigated here exhibit bistability, i.e. the solution realized depends on the initial conditions for the magnetic field. We started most of the simulations with a dipolar magnetic field, but varied its initial amplitude over several orders of magnitude. Some calculations were started from another model with slightly different parameters to test for hysteresis. In the bistable regime, models resulting from simulations with an initially weak magnetic field are here referred to as the 'non-dipolar branch'. They are distinguished from dipolar solutions initially started with a strong magnetic field.

The numerical solver used to compute solutions of equations (11)-(3) is PaRoDy (Dormy et al. (1998) and further developments). The numerical method is similar to that described in Glatzmaier (1984) except for the radial discretisation, which is treated in physical space on a stretched grid (allowing for a parallelization by a radial domain decomposition). Moreover, the pressure term has been eliminated by considering the double curl of the momentum equation.

\section{Non-Dimensional Output Parameters}

Our numerical dynamo-models are characterized by non-dimensional output parameters. Dimensionless measures for the flow velocity are the magnetic Reynolds number, $R m=$

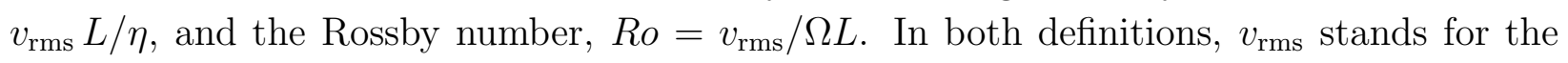
rms velocity of the flow. Similarly, $B_{\text {rms }}$ denotes the rms field strength. We also introduce a local Rossby number, $R o_{\ell}=R o \bar{\ell}_{p} / \pi$, based on the mean harmonic degree $\bar{\ell}_{p}$ of the poloidal velocity field,

$$
\bar{\ell}_{p}=\sum_{\ell} \ell \frac{<\left(\boldsymbol{v}_{\boldsymbol{p}}\right)_{\ell} \cdot\left(\boldsymbol{v}_{\boldsymbol{p}}\right)_{\ell}>}{<\boldsymbol{v}_{\boldsymbol{p}} \cdot \boldsymbol{v}_{\boldsymbol{p}}>} .
$$

The brackets in (4) denote an average over time and radii. In contrast to the definition introduced by Christensen \& Aubert (2006), our modified Rossby number relies on a convective 
length-scale and not on the mean half wavelength of the total flow (see also the Appendix).

The magnetic field strength is measured by the dimensionless Lorentz number, Lo = $B_{\mathrm{rms}} /(\sqrt{\varrho \mu} \Omega L)$, and the classical Elsasser number $\Lambda=B_{\mathrm{rms}}^{2} / \Omega \varrho \mu \eta$. They are related through

$\Lambda=L o^{2} \mathrm{Pm} / \mathrm{E}$. Moreover, following Christensen \& Aubert (2006), we characterize the geometry of the magnetic field by the relative dipole field strength, $f_{\text {dip }}$, which is defined as the ratio of the average field strength of the dipole field to the field strength in harmonic degrees $\ell=1, \cdots, 12$ at the outer boundary.

A non-dimensional measure for the heat transport is given by the Nusselt number, $N u$, defined as the ratio of the total heat flow to the conducted heat flow, $Q_{\text {cond }}=4 \pi r_{o} r_{i} \varrho c \kappa \Delta T / L$ with the heat capacity $c$.

\section{Mean-Field Analysis}

We present dynamo models obtained by direct numerical simulations (DNS) and do not use the mean-field formalism (Steenbeck et al. 1966; Moffatt 1978; Krause \& Rädler 1980) to predict any dynamo action. However, the mean-field approach provides useful theoretical concepts and mathematical tools to interpret numerical dynamo models. It is usually set up by splitting the velocity and the magnetic field in mean and fluctuating parts, $\boldsymbol{v}=\overline{\boldsymbol{V}}+\boldsymbol{v}^{\prime}$ and $\boldsymbol{B}=\overline{\boldsymbol{B}}+\boldsymbol{b}^{\prime}$. Mean quantities are here denoted by an overbar and defined as azimuthal averages. The objective of mean-field theory is to predict the evolution of the averaged or mean magnetic field, $\overline{\boldsymbol{B}}$, which is in general determined by the dynamo equation,

$$
\frac{\partial \overline{\boldsymbol{B}}}{\partial t}=\nabla \times(\mathcal{E}+\overline{\boldsymbol{V}} \times \overline{\boldsymbol{B}}-\eta \nabla \times \overline{\boldsymbol{B}}) .
$$

The crucial new term in (5) compared to the induction equation is the mean electromotive force, $\mathcal{E}=\overline{\boldsymbol{v}^{\prime} \times \boldsymbol{b}^{\prime}}$. It is a functional of $\boldsymbol{v}, \overline{\boldsymbol{V}}$, and $\overline{\boldsymbol{B}}$, which is affin-linear in $\overline{\boldsymbol{B}}$. Provided that there is no small-scale dynamo action, $\mathcal{E}$ is also homogeneous in $\overline{\boldsymbol{B}}$. If, moreover, the mean electromotive force depends only instantaneously and locally on $\overline{\boldsymbol{B}}$, it may be parameterized in terms of the mean field and its first derivatives,

$$
\mathcal{E}=-\boldsymbol{\alpha} \overline{\boldsymbol{B}}-\boldsymbol{\gamma} \times \overline{\boldsymbol{B}}-\boldsymbol{\beta}(\nabla \times \overline{\boldsymbol{B}})-\boldsymbol{\delta} \times(\nabla \times \overline{\boldsymbol{B}})-\boldsymbol{\kappa} \nabla \overline{\boldsymbol{B}}
$$

The parameters, also known as mean-field coefficients, are vectors $(\boldsymbol{\gamma}$ and $\boldsymbol{\delta})$, symmetric tensors of second rank $(\boldsymbol{\alpha}$ and $\boldsymbol{\beta}$ ) and a third rank tensor $(\boldsymbol{\kappa})$. They depend only on the velocity field and the magnetic diffusivity, but not (explicitly) on the magnetic field. A physical interpretation of the mean-field coefficients is given by Rädler (1995). The $\alpha$-tensor describes the classical $\alpha$-effect (see also Parker 1955), $\gamma$ is associated with an advective 
transport of the mean magnetic field, $\beta$ can be interpreted as a turbulent diffusivity, $\delta$ may contribute to a shear-current effect, an inductive effect first noted by Rädler (1969a, b $)$, whereas the $\kappa$-terms are more difficult to interpret. The altogether 27 independent meanfield coefficients were determined for several numerical models with the help of the so-called test-field method (Schrinner et al. 2005, 2007).

A comparison between DNS and mean-field calculations revealed that parameterization (6) is indeed reliable for a wide class of dynamo models (Schrinner 2011; Schrinner et al. 2011). An important limitation is the intrinsic kinematic character of the mean-field approach followed here. In general, it is therefore only applicable to dynamo models which can be reproduced kinematically and belong to the so-called kinematically stable regime identified by Schrinner et al. (2010a).

For a mean-field analysis of some of our dynamo models derived from (11)-(3), we solve the dynamo equation as an eigenvalue problem

$$
\sigma \overline{\boldsymbol{B}}=\nabla \times \boldsymbol{D} \overline{\boldsymbol{B}}
$$

with the time-averaged dynamo operator $\boldsymbol{D}$ defined through

$$
\boldsymbol{D} \overline{\boldsymbol{B}}=\overline{\boldsymbol{v}} \times \overline{\boldsymbol{B}}-\boldsymbol{\alpha} \overline{\boldsymbol{B}}-\boldsymbol{\gamma} \times \overline{\boldsymbol{B}}-\boldsymbol{\beta}(\nabla \times \overline{\boldsymbol{B}})-\boldsymbol{\delta} \times(\nabla \times \overline{\boldsymbol{B}})-\boldsymbol{\kappa} \nabla \overline{\boldsymbol{B}} .
$$

On average, the evolution of the mean field is then proportional to $\boldsymbol{b}^{i} \exp \left(\sigma_{i} t\right)$ with eigenvectors $\boldsymbol{b}^{i}$ and eigenvalues $\sigma_{i}$. More details about the eigenvalue calculation are given in Schrinner et al. (2010b).

\section{Results}

We present results from 72 dynamo models obtained by varying all four dimensionless control parameters, the aspect ratio of the shell as well as the mechanical boundary conditions. Table 1 is divided in three sections for three different types of mechanical boundary conditions and provides the control parameters and the output parameters introduced above for each model. Within each section, the models are listed in the order of increasing local Rossby number. For some models with stress-free mechanical boundary conditions, two different solutions coexist depending on the initial conditions for the magnetic field. Thus, these models appear in pairs and are labeled by the letter ' $d$ ' (dipolar) if the initial magnetic field was strong and by 'm' (multipolar) if the simulations were started from a weak magnetic field.

Kutzner \& Christensen (2002) identified a dipolar and a multipolar dynamo regime. Both regimes can be recovered here if the models are ordered by the local Rossby number 
introduced in section 3 (see also Christensen \& Aubert 2006). Figure 1 (left panel) shows two distinct dynamo regimes: models with a dipole dominated magnetic field at low Rossby numbers (filled symbols) and models with a more multipolar magnetic field at higher Rossby numbers (open symbols). A fairly sharp transition between both regimes occurs at $R o_{\ell} \approx 0.1$. The exact value may depend weakly on the choice of the mechanical boundary conditions and seems to be closer to $R_{\ell} \approx 0.12$ for models with rigid boundaries. The right panel of Fig. 1 illustrates that models of both regimes differ in their field topology, but not necessarily in their field strength. We emphasize that only the definition of a local Rossby number based on a convective length scale (i.e. not taking into account the zonal flow) enables us to extend the well known dipole-multipole partition to models with different aspect ratios and boundary conditions. This is most clearly demonstrated by the sequence of models 29,31 , 32, 34 and 35 from Table 1 which is depicted in Fig. 2, For these models, the Ekman number, the Rayleigh number (normalized by its critical value), and the Prandtl numbers were kept constant whereas the aspect ratio was progressively increased. The thinner the convection zone, the smaller the convective length scales, and the mean harmonic degree of the poloidal velocity field increases systematically from $\bar{\ell}_{p}=11$ for model 29 with $\chi=0.5$ to $\bar{\ell}_{p}=20$ for model 35 with $\chi=0.65$. Consequently, $R o_{\ell}$ grows from $R o_{\ell}=4.70 \times 10^{-2}$ for model 29 to $R o_{\ell}=1.14 \times 10^{-1}$ for model 35 , which already belongs to the multipolar regime. The decrease of the convective length scales caused a transition towards the multipolar regime and is adequately measured by the local Rossby number introduced here. If, however, $\bar{\ell}_{p}$ were derived from the total velocity field, neither $\bar{\ell}_{p}$ nor $R o_{\ell}$ would increase in the same way, owing to a major contribution of the mean zonal flow to the kinetic energy density in model 35. The Rossby number criterion would then fail to predict the observed transition between both dynamo regimes. Note that decreasing the Ekman number from $E=10^{-3}$ to $E=310^{-4}$ in model 30 leads again to a dipolar field despite the high aspect ratio of $\chi=0.65$ and that we find a multipolar magnetic field for model 37 at lower aspect ratio, $\chi=0.6$, but higher Rayleigh number. Both examples illustrate that the transition is indeed characterized by $R o_{\ell}$. However, the definition of $R o_{\ell}$ is empirically motivated, there may be other ways to define a relevant Rossby number to distinguish both dynamo regimes; some of them are discussed in Appendix A.

Moreover, Fig. 3 demonstrates that the relative dipole field strength does not simply depend on the magnetic Reynolds number. The distance from the dynamo threshold of a model does not determine its field topology.

An exception to the Rossby number criterion for the dipolarity of the magnetic field in dynamo models occurs in the case of stress free boundary conditions. Simulations started from a weak magnetic field (m-models) are not simply dipole dominated, independent of their local Rossby number. These models left out in Fig. 1 were included in Fig. 4 and 

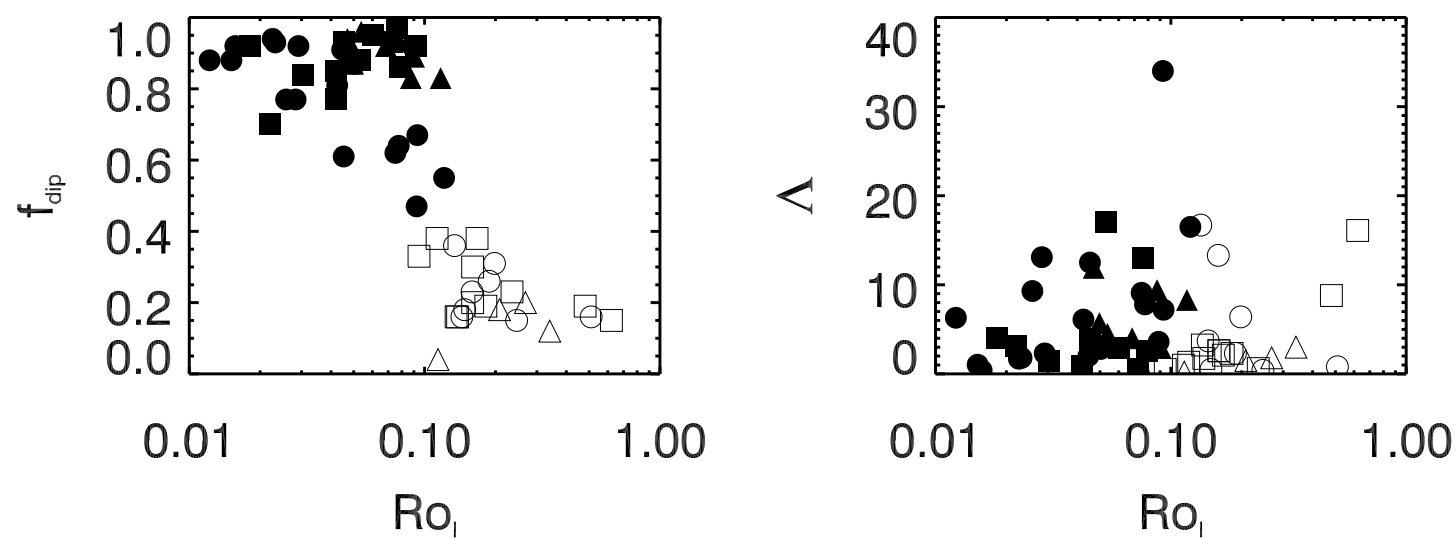

Fig. 1. - Left: Relative dipole field strength versus the local Rossby number for all models from Table 1 apart from those with stress-free boundary conditions belonging to the multipolar branch (m-models). Filled symbols stand for models dominated by a dipole field, open symbols denote multipolar models. The symbol shape indicates different types of mechanical boundary conditions: circles mean no-slip conditions at both boundaries, triangles are models with a rigid inner and and a stress-free outer boundary, and squares stand for models with stress-free conditions at both boundaries. Right: Elsasser number versus local Rossby number for the same sample of models. 
are represented by diamonds. As apparent from Fig. 4, dynamo models with stress free mechanical boundary conditions form two branches, an upper branch of dipolar models for $R o_{\ell}<0.1$ and a lower branch of models with a less constrained field geometry (see also Busse \& Simitev 2009; Simitev \& Busse 2009). We refer to them as the 'dipolar' and the 'multipolar' branch instead of the 'weak-field' and the 'strong-field' branch to avoid confusion with corresponding notions introduced in the limit of vanishing viscosity and inertia in the context of planetary dynamos. Nevertheless, the saturated magnetic field strength, as measured by the Lorentz number, is always larger for dipolar than for multipolar models. For $R o_{\ell}>0.1$, both branches coincide and their distinction is no longer meaningful (see Fig (14). The region of bistability in parameter space, however, does not solely depend on the local Rossby number, but also on the magnetic Prandtl number (Busse \& Simitev 2009); for models 40, 42, and 44, a weak-field branch does not exist.

There is a strong correlation between the topology and the time dependence of the magnetic field in dynamo models. Sudden polarity reversals or oscillations of the magnetic field do not occur in dipole dominated models in the low Rossby number regime. Conversely, reversals and oscillations are frequent in non-dipolar models with $R o_{\ell}>0.1$ as well as in models with lower local Rossby numbers with stress-free boundary conditions belonging to the multipolar branch. Whether non-dipolar models exhibit fairly coherent oscillations or irregular reversals of the magnetic field strongly depends on the magnetic Reynolds number. Coherent oscillatory solutions of the induction equation are most clearly visible in so-called butterfly diagrams; contours of the azimuthally averaged radial magnetic field at the outer boundary are plotted versus time and colatitude. Figure 5 gives two examples. The left panel shows a very coherent dynamo wave at $R m=102$ (model $45 \mathrm{~m}$ ), whereas the butterfly diagram at $R m=258$ on the right-hand side (model 38) is much less periodic and a cycle period cannot be identified. Dynamo models (in the non-dipolar regime) at higher magnetic Reynolds number exhibit even less temporal coherence. Following this somewhat arbitrary and qualitative criterion, we find that non-dipolar dynamos of our sample with $R m \lesssim 200$ generate magnetic fields which vary periodically in time. The lower the magnetic Reynolds number, the more coherent is the time variability of the magnetic field. A third property is intimately linked to the topology and the time dependence of the magnetic field. Schrinner et al. (2010a) looked at the evolution of a passive vector field described by a second induction equation which was solved simultaneously with equations (11)-(3). The magnetic 'tracer-field' was advanced at each time-step with the self-consistently determined velocity field but did not contribute to the Lorentz force. A similar experiment had been performed by Cattaneo \& Tobias (2009) and Tilgner \& Brandenburg (2008), too. Schrinner et al. (2010a) found that the tracer field grows exponentially for multipolar and reversing models, whereas it remains kinematically stable for dipolar dynamos in the low Rossby number regime. This 

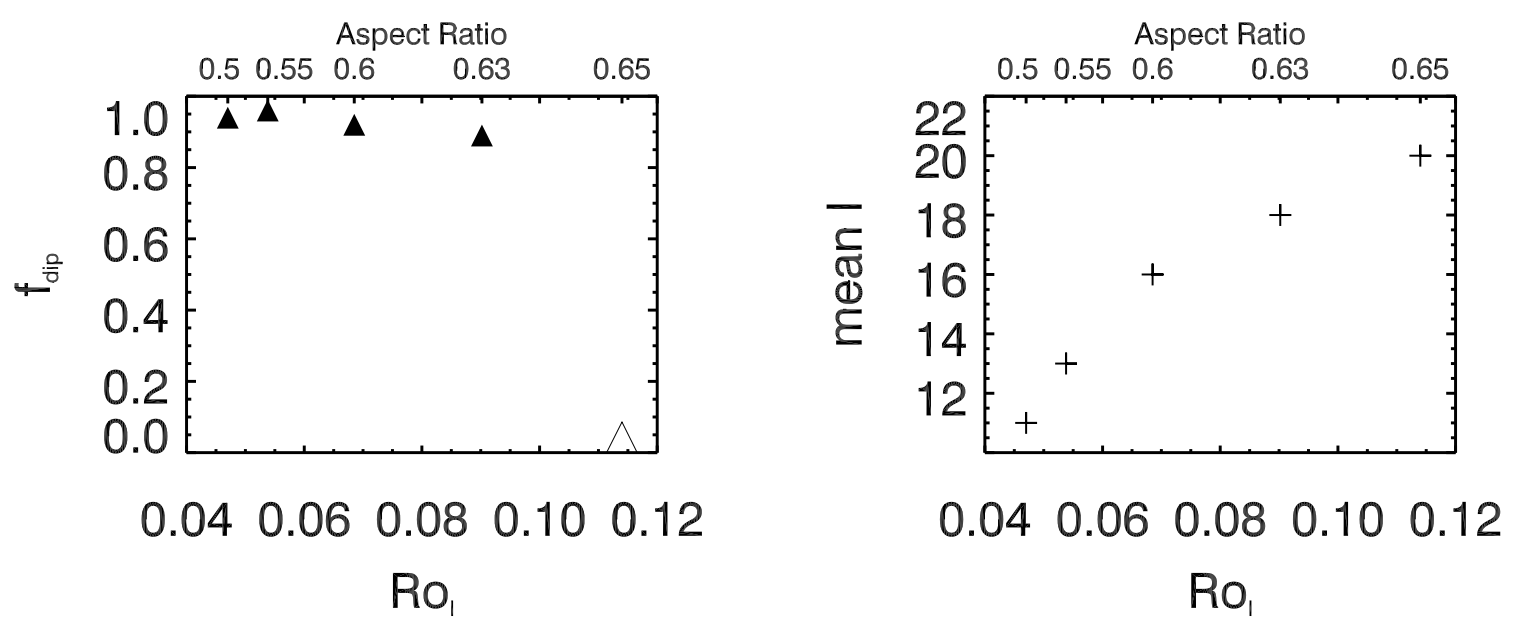

Fig. 2.- The relative dipole field strength for models 29, 31, 32, 34 (filled triangles) and model 35 (open triangle) and the mean harmonic degree $\bar{\ell}_{p}$ of the poloidal velocity field (right panel) versus the local Rossby number. The aspect ratio (upper x-axis) has been gradually increased for this sequence of models leading to larger $\bar{\ell}_{p}$ and thus also to larger $R_{\ell}$. Model 35 has undergone a transition from the dipolar to the multipolar dynamo regime and the dipole field strength has dropped drastically.

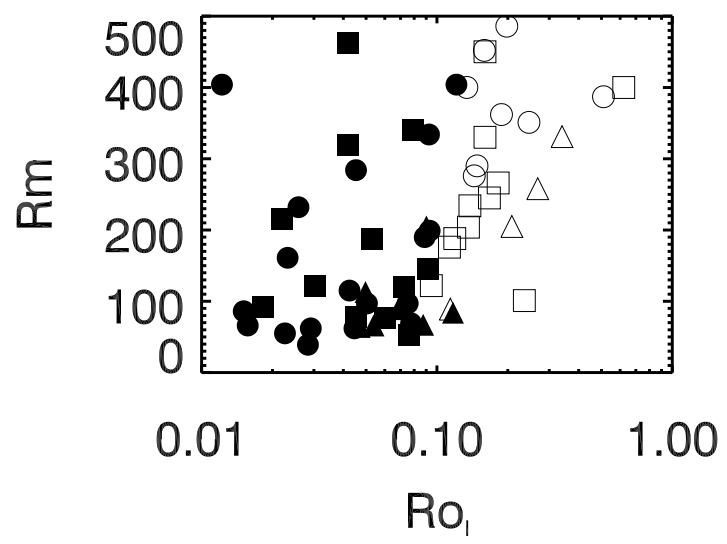

Fig. 3.- The magnetic Reynolds number versus the local Rossby number for all models from Table 1. For the meaning of the symbol style we refer to the caption of Fig. 1. 


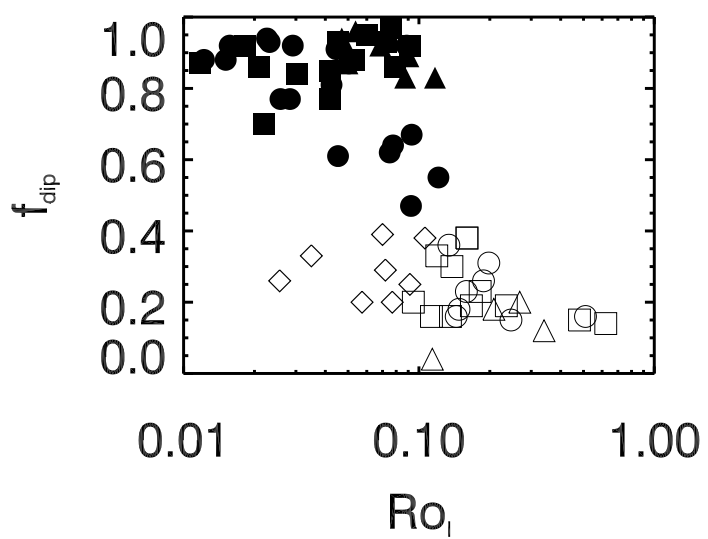

Fig. 4.- The relative dipole field strength versus the local Rossby number as in Fig. 1 but here with the m-models included (diamonds). All other notations as in Fig. 1.

relation can be extended to the sample of models studied here and serves to distinguish both dynamo classes. Dipolar models represented by filled symbols in Fig. 4 are kinematically stable, all multipolar models represented by open symbols are kinematically unstable. 
Table 1. Overview of the simulations carried out, ordered with respect to their modified Rossby number and their mechanical boundary conditions.

\begin{tabular}{|c|c|c|c|c|c|c|c|c|c|c|}
\hline Model & $E$ & $R a$ & $\mathrm{Pm}$ & $\operatorname{Pr}$ & $\chi$ & $\bar{\ell}_{p}$ & $R o_{\ell}$ & $f_{\text {dip }}$ & $R m$ & Lo \\
\hline \multicolumn{11}{|c|}{ Rigid Boundary Conditions } \\
\hline 1 & $3 \times 10^{-5}$ & 510 & 3 & 1 & 0.35 & 14 & $7.37 \times 10^{-3}$ & 0.88 & 161 & $4.92 \times 10^{-3}$ \\
\hline 2 & $3 \times 10^{-5}$ & 510 & 4 & 1 & 0.35 & 14 & $7.88 \times 10^{-3}$ & 0.91 & 232 & $8.02 \times 10^{-3}$ \\
\hline 3 & $1 \times 10^{-3}$ & 100 & 5 & 1 & 0.35 & 5 & $1.22 \times 10^{-2}$ & 0.88 & 39 & $3.54 \times 10^{-2}$ \\
\hline 4 & $1 \times 10^{-4}$ & 334 & 2 & 1 & 0.35 & 11 & $1.51 \times 10^{-2}$ & 0.88 & 86 & $7.18 \times 10^{-3}$ \\
\hline 5 & $3 \times 10^{-4}$ & 195 & 3 & 1 & 0.35 & 8 & $1.57 \times 10^{-2}$ & 0.92 & 66 & $6.53 \times 10^{-3}$ \\
\hline 6 & $3 \times 10^{-4}$ & 243 & 2 & 1 & 0.35 & 9 & $2.26 \times 10^{-2}$ & 0.94 & 55 & $1.61 \times 10^{-3}$ \\
\hline 7 & $3 \times 10^{-5}$ & 750 & 1 & 1 & 0.35 & 25 & $2.32 \times 10^{-2}$ & 0.93 & 97 & $7.35 \times 10^{-2}$ \\
\hline 8 & $1 \times 10^{-3}$ & 150 & 5 & 1 & 0.40 & 6 & $2.58 \times 10^{-2}$ & 0.77 & 69 & $4.32 \times 10^{-3}$ \\
\hline 9 & $1 \times 10^{-3}$ & 136 & 5 & 1 & 0.45 & 7 & $2.83 \times 10^{-2}$ & 0.77 & 62 & $5.11 \times 10^{-2}$ \\
\hline 10 & $3 \times 10^{-4}$ & 285 & 2 & 1 & 0.35 & 10 & $2.91 \times 10^{-2}$ & 0.92 & 62 & $1.84 \times 10^{-2}$ \\
\hline 11 & $3 \times 10^{-4}$ & 375 & 3 & 1 & 0.35 & 12 & $4.25 \times 10^{-2}$ & 0.81 & 115 & $2.47 \times 10^{-2}$ \\
\hline 12 & $3 \times 10^{-4}$ & 375 & 1.5 & 1 & 0.35 & 11 & $4.46 \times 10^{-2}$ & 0.91 & 62 & $1.98 \times 10^{-2}$ \\
\hline 13 & $3 \times 10^{-4}$ & 330 & 9 & 3 & 0.35 & 15 & $4.53 \times 10^{-2}$ & 0.61 & 284 & $2.04 \times 10^{-2}$ \\
\hline 14 & $3 \times 10^{-4}$ & 330 & 3 & 3 & 0.35 & 16 & $5.04 \times 10^{-2}$ & 0.87 & 97 & $1.69 \times 10^{-2}$ \\
\hline 15 & $1 \times 10^{-3}$ & 100 & 7 & 1 & 0.65 & 16 & $7.51 \times 10^{-2}$ & 0.62 & 107 & $3.60 \times 10^{-2}$ \\
\hline 16 & $1 \times 10^{-3}$ & 100 & 6 & 1 & 0.65 & 16 & $7.76 \times 10^{-2}$ & 0.64 & 93 & $3.61 \times 10^{-2}$ \\
\hline 17 & $1 \times 10^{-4}$ & 1117 & 1.5 & 0.67 & 0.35 & 22 & $8.87 \times 10^{-2}$ & 0.92 & 190 & $1.54 \times 10^{-2}$ \\
\hline 18 & $1 \times 10^{-3}$ & 400 & 10 & 1 & 0.35 & 9 & $9.25 \times 10^{-2}$ & 0.47 & 334 & $5.81 \times 10^{-2}$ \\
\hline 19 & $3 \times 10^{-4}$ & 630 & 3 & 1 & 0.35 & 15 & $9.31 \times 10^{-2}$ & 0.67 & 199 & $2.68 \times 10^{-2}$ \\
\hline 20 & $3 \times 10^{-4}$ & 810 & 5 & 1 & 0.35 & 16 & $1.21 \times 10^{-1}$ & 0.55 & 404 & $3.15 \times 10^{-2}$ \\
\hline 21 & $1 \times 10^{-3}$ & 450 & 10 & 1 & 0.35 & 11 & $1.34 \times 10^{-1}$ & 0.36 & 401 & $4.08 \times 10^{-2}$ \\
\hline 22 & $3 \times 10^{-4}$ & 810 & 3 & 1 & 0.35 & 16 & $1.44 \times 10^{-1}$ & 0.16 & 277 & $1.93 \times 10^{-2}$ \\
\hline 23 & $3 \times 10^{-4}$ & 750 & 3 & 1 & 0.35 & 16 & $1.48 \times 10^{-1}$ & 0.18 & 290 & $7.14 \times 10^{-2}$ \\
\hline 24 & $1 \times 10^{-3}$ & 500 & 10 & 1 & 0.35 & 11 & $1.59 \times 10^{-1}$ & 0.23 & 453 & $3.65 \times 10^{-3}$ \\
\hline 25 & $3 \times 10^{-4}$ & 1050 & 3 & 1 & 0.35 & 16 & $1.88 \times 10^{-1}$ & 0.26 & 363 & $1.52 \times 10^{-2}$ \\
\hline 26 & $3 \times 10^{-4}$ & 1250 & 3 & 0.3 & 0.35 & 13 & $1.98 \times 10^{-1}$ & 0.31 & 486 & $2.52 \times 10^{-2}$ \\
\hline 27 & $1 \times 10^{-4}$ & 1117 & 1.5 & 0.67 & 0.5 & 33 & $2.46 \times 10^{-1}$ & 0.15 & 351 & $5.06 \times 10^{-3}$ \\
\hline 28 & $3 \times 10^{-4}$ & 2970 & 1 & 0.3 & 0.35 & 14 & $5.10 \times 10^{-1}$ & 0.16 & 387 & $1.55 \times 10^{-2}$ \\
\hline \multicolumn{11}{|c|}{ Mixed Boundary Conditions } \\
\hline 29 & $1 \times 10^{-3}$ & 125 & 5 & 1 & 0.50 & 11 & $4.70 \times 10^{-2}$ & 0.94 & 67 & $4.88 \times 10^{-2}$ \\
\hline 30 & $3 \times 10^{-4}$ & 120 & 5 & 1 & 0.65 & 26 & $4.97 \times 10^{-2}$ & 0.87 & 100 & $1.86 \times 10^{-2}$ \\
\hline 31 & $1 \times 10^{-3}$ & 110 & 5 & 1 & 0.55 & 13 & $5.38 \times 10^{-2}$ & 0.96 & 64 & $2.99 \times 10^{-2}$ \\
\hline 32 & $1 \times 10^{-3}$ & 105 & 5 & 1 & 0.60 & 16 & $6.85 \times 10^{-2}$ & 0.92 & 66 & $2.84 \times 10^{-2}$ \\
\hline 33 & $1 \times 10^{-3}$ & 125 & 5 & 1 & 0.60 & 16 & $8.74 \times 10^{-2}$ & 0.83 & 84 & $4.34 \times 10^{-2}$ \\
\hline 34 & $1 \times 10^{-3}$ & 105 & 5 & 1 & 0.63 & 18 & $9.02 \times 10^{-2}$ & 0.89 & 78 & $2.47 \times 10^{-2}$ \\
\hline 35 & $1 \times 10^{-3}$ & 100 & 5 & 1 & 0.65 & 20 & $1.14 \times 10^{-1}$ & 0.04 & 89 & $6.63 \times 10^{-3}$ \\
\hline 36 & $1 \times 10^{-3}$ & 150 & 5 & 1 & 0.60 & 16 & $1.17 \times 10^{-1}$ & 0.83 & 113 & $4.09 \times 10^{-2}$ \\
\hline 37 & $1 \times 10^{-3}$ & 175 & 5 & 1 & 0.60 & 16 & $2.08 \times 10^{-1}$ & 0.18 & 205 & $1.71 \times 10^{-2}$ \\
\hline 38 & $1 \times 10^{-3}$ & 200 & 5 & 1 & 0.60 & 16 & $2.68 \times 10^{-1}$ & 0.20 & 258 & $1.87 \times 10^{-2}$ \\
\hline 39 & $1 \times 10^{-3}$ & 250 & 5 & 1 & 0.60 & 16 & $3.40 \times 10^{-1}$ & 0.12 & 331 & $2.55 \times 10^{-2}$ \\
\hline \multicolumn{11}{|c|}{ Stress-Free Boundary Conditions } \\
\hline 40 & $1 \times 10^{-4}$ & 365 & 2 & 1 & 0.35 & 12 & $1.82 \times 10^{-2}$ & 0.92 & 92 & $5.69 \times 10^{-3}$ \\
\hline $41 \mathrm{~d}$ & $3 \times 10^{-5}$ & 600 & 1 & 1 & 0.35 & 16 & $2.11 \times 10^{-2}$ & 0.86 & 140 & $3.82 \times 10^{-3}$ \\
\hline $41 \mathrm{~m}$ & $3 \times 10^{-5}$ & 600 & 1 & 1 & 0.35 & 16 & $2.56 \times 10^{-2}$ & 0.26 & 144 & $2.35 \times 10^{-3}$ \\
\hline 42 & $1 \times 10^{-4}$ & 375 & 4 & 1 & 0.35 & 13 & $2.20 \times 10^{-2}$ & 0.70 & 216 & $8.80 \times 10^{-3}$ \\
\hline
\end{tabular}


Table 1-Continued

\begin{tabular}{|c|c|c|c|c|c|c|c|c|c|c|}
\hline Model & $E$ & $R a$ & $P m$ & $\operatorname{Pr}$ & $\chi$ & $\bar{\ell}_{p}$ & $R o_{\ell}$ & $f_{\text {dip }}$ & $R m$ & Lo \\
\hline $43 \mathrm{~d}$ & $1 \times 10^{-4}$ & 462 & 2 & 1 & 0.35 & 16 & $3.04 \times 10^{-2}$ & 0.84 & 121 & $8.44 \times 10^{-3}$ \\
\hline $43 \mathrm{~m}$ & $1 \times 10^{-4}$ & 462 & 2 & 1 & 0.35 & 16 & $3.49 \times 10^{-2}$ & 0.33 & 146 & $4.91 \times 10^{-3}$ \\
\hline 44 & $1 \times 10^{-4}$ & 750 & 6 & 1 & 0.35 & 17 & $4.20 \times 10^{-2}$ & 0.77 & 462 & $2.50 \times 10^{-2}$ \\
\hline $45 \mathrm{~d}$ & $1 \times 10^{-4}$ & 586 & 1 & 1 & 0.35 & 18 & $4.54 \times 10^{-2}$ & 0.93 & 78 & $9.71 \times 10^{-3}$ \\
\hline $45 \mathrm{~m}$ & $1 \times 10^{-4}$ & 586 & 1 & 1 & 0.35 & 18 & $5.73 \times 10^{-2}$ & 0.20 & 102 & $5.60 \times 10^{-3}$ \\
\hline $46 \mathrm{~d}$ & $1 \times 10^{-4}$ & 749 & 2 & 1 & 0.35 & 18 & $5.30 \times 10^{-2}$ & 0.88 & 187 & $1.40 \times 10^{-2}$ \\
\hline $46 \mathrm{~m}$ & $1 \times 10^{-4}$ & 749 & 2 & 1 & 0.35 & 18 & $7.00 \times 10^{-2}$ & 0.39 & 244 & $8.12 \times 10^{-3}$ \\
\hline $47 \mathrm{~d}$ & $1 \times 10^{-4}$ & 750 & 4 & 1 & 0.35 & 17 & $4.20 \times 10^{-2}$ & 0.85 & 320 & $2.06 \times 10^{-2}$ \\
\hline $47 \mathrm{~m}$ & $1 \times 10^{-4}$ & 750 & 4 & 1 & 0.35 & 20 & $7.20 \times 10^{-2}$ & 0.29 & 460 & $9.89 \times 10^{-3}$ \\
\hline $48 \mathrm{~d}$ & $1 \times 10^{-4}$ & 750 & 0.75 & 1 & 0.35 & 19 & $6.03 \times 10^{-2}$ & 0.95 & 77 & $1.11 \times 10^{-2}$ \\
\hline $48 \mathrm{~m}$ & $1 \times 10^{-4}$ & 750 & 0.75 & 1 & 0.35 & 18 & $7.70 \times 10^{-2}$ & 0.20 & 100 & $7.02 \times 10^{-3}$ \\
\hline $49 \mathrm{~d}$ & $3 \times 10^{-4}$ & 510 & 2 & 1 & 0.35 & 13 & $7.26 \times 10^{-2}$ & 0.93 & 120 & $2.08 \times 10^{-2}$ \\
\hline $49 \mathrm{~m}$ & $3 \times 10^{-4}$ & 510 & 2 & 1 & 0.35 & 12 & $9.15 \times 10^{-2}$ & 0.25 & 155 & $1.09 \times 10^{-2}$ \\
\hline $50 \mathrm{~d}$ & $1 \times 10^{-4}$ & 750 & 0.5 & 1 & 0.35 & 12 & $7.60 \times 10^{-2}$ & 0.97 & 53 & $1.03 \times 10^{-2}$ \\
\hline $50 \mathrm{~m}$ & $1 \times 10^{-4}$ & 750 & 0.5 & 1 & 0.35 & 12 & $8.20 \times 10^{-2}$ & 0.21 & 70 & $6.32 \times 10^{-3}$ \\
\hline $51 \mathrm{~d}$ & $1 \times 10^{-4}$ & 750 & 2 & 0.3 & 0.35 & 15 & $7.90 \times 10^{-2}$ & 0.86 & 340 & $2.55 \times 10^{-2}$ \\
\hline $51 \mathrm{~m}$ & $1 \times 10^{-4}$ & 750 & 2 & 0.3 & 0.35 & 15 & $1.06 \times 10^{-1}$ & 0.38 & 450 & $1.52 \times 10^{-2}$ \\
\hline $52 \mathrm{~d}$ & $1 \times 10^{-4}$ & 1110 & 1 & 1 & 0.35 & 20 & $9.20 \times 10^{-2}$ & 0.92 & 146 & $1.61 \times 10^{-2}$ \\
\hline $52 \mathrm{~m}$ & $1 \times 10^{-4}$ & 1110 & 1 & 1 & 0.35 & 20 & $1.19 \times 10^{-1}$ & 0.33 & 188 & $1.13 \times 10^{-2}$ \\
\hline 53 & $3 \times 10^{-4}$ & 510 & 1.5 & 1 & 0.35 & 12 & $9.46 \times 10^{-2}$ & 0.20 & 122 & $1.01 \times 10^{-2}$ \\
\hline 54 & $1 \times 10^{-4}$ & 1333 & 2 & 0.3 & 0.35 & 15 & $1.60 \times 10^{-1}$ & 0.38 & 450 & $1.52 \times 10^{-2}$ \\
\hline 55 & $1 \times 10^{-4}$ & 1000 & 1 & 1 & 0.35 & 20 & $1.13 \times 10^{-1}$ & 0.16 & 176 & $9.83 \times 10^{-3}$ \\
\hline 56 & $1 \times 10^{-4}$ & 1200 & 1 & 1 & 0.35 & 21 & $1.36 \times 10^{-1}$ & 0.16 & 204 & $1.18 \times 10^{-2}$ \\
\hline 57 & $1 \times 10^{-4}$ & 1372 & 1 & 1 & 0.35 & 19 & $1.38 \times 10^{-1}$ & 0.30 & 234 & $1.32 \times 10^{-2}$ \\
\hline 58 & $1 \times 10^{-4}$ & 1757 & 1 & 0.3 & 0.35 & 15 & $1.60 \times 10^{-1}$ & 0.38 & 330 & $1.61 \times 10^{-2}$ \\
\hline 59 & $1 \times 10^{-4}$ & 1497 & 1 & 1 & 0.35 & 21 & $1.67 \times 10^{-1}$ & 0.19 & 245 & $1.45 \times 10^{-2}$ \\
\hline 60 & $1 \times 10^{-4}$ & 1627 & 1 & 1 & 0.35 & 22 & $1.82 \times 10^{-1}$ & 0.23 & 266 & $1.52 \times 10^{-2}$ \\
\hline 61 & $1 \times 10^{-4}$ & 1823 & 0.3 & 1 & 0.35 & 22 & $2.35 \times 10^{-1}$ & 0.19 & 101 & $1.45 \times 10^{-2}$ \\
\hline 62 & $3 \times 10^{-4}$ & 2000 & 2 & 0.3 & 0.35 & 15 & $4.81 \times 10^{-1}$ & 0.15 & 658 & $3.63 \times 10^{-2}$ \\
\hline
\end{tabular}

Note. - Some of the models in Table 1 were already published in Schrinner et al. (2010a) with a somewhat different definition of $\bar{\ell}_{p}$ and thus also of $R o_{\ell}$. 

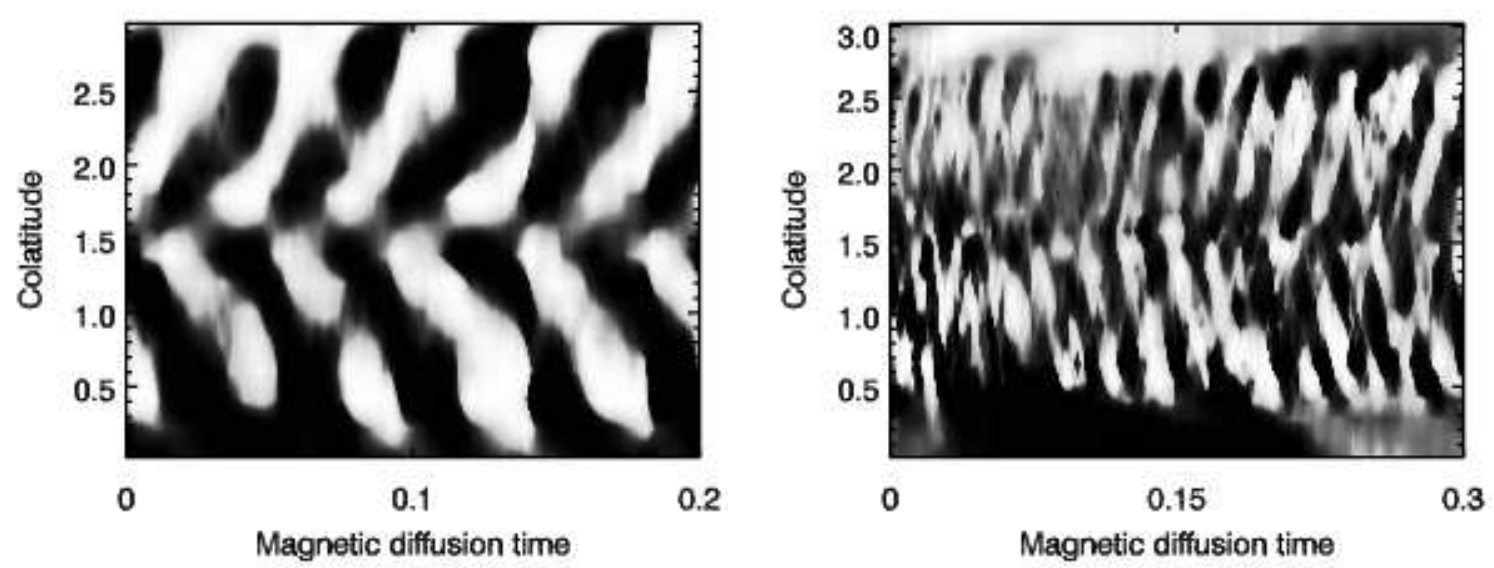

Fig. 5.- Left panel: Butterfly diagram for model $45 \mathrm{~m}, R m=102$. Right panel: Butterfly diagram for model $38, R m=258$. The contour plots are normalized by their maximum absolute value and the grey-scale coding varies from +1 (black) to -1 (white).

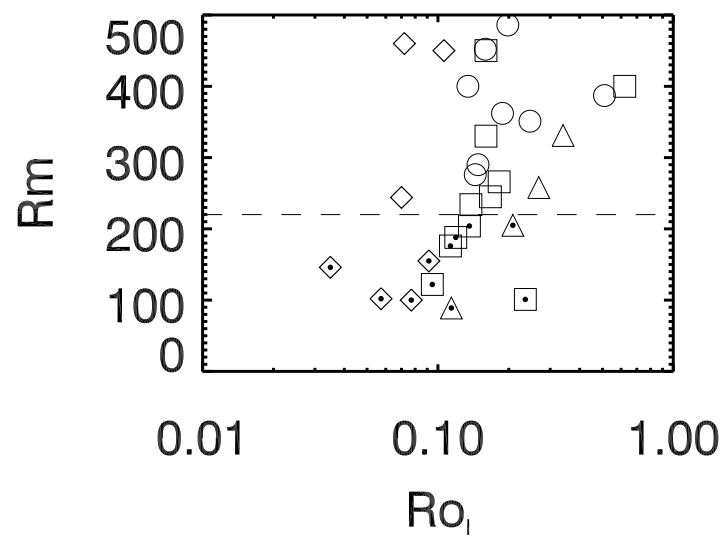

Fig. 6.- Magnetic Reynolds number versus the local Rossby number for all multipolar models of Table 1. A dot in an open symbol indicates that a coherent dynamo wave was found. The meaning of all other notations is as defined in Fig. 1 and Fig. 4. 


\section{Discussion}

\subsection{Reason for the dipole breakdown}

What causes the dipolarity of dynamo models far above the dynamo threshold in the low Rossby number regime? The Rossby number criterion supported by this work predicts a dipole dominated magnetic field, if the typical length scale of convection, $\left(\bar{\ell}_{p} / \pi\right) L$, is at least one order of magnitude larger than the Rossby radius, i.e. $0.1\left(\bar{\ell}_{p} / \pi\right) L>v_{\text {rms }} / \Omega$. Convection is then strongly influenced by rotation and organized in columns parallel to the rotation axis. A mean-field analysis reveals that the magnetic field generated and maintained by these convective rolls is dominated by only one real dipolar eigenmode with approximately zero growth rate. All more structured and in general complex overtones (i.e. higher order modes) are highly diffusive. The kinematic stability of models at low $R o_{\ell}$ as well as their comparatively steady dipole field are a consequence of this single mode property (Schrinner et al. 2010a). However, the reason for the breakdown of the dipole field at $R o_{\ell} \approx 0.1$ is at present not well understood. We computed the eigenvalue spectra and the eigenmodes of the time-averaged dynamo operator $D$ for a sequence of models with increasing $R o_{\ell}$ to gain more insight. The sequence consists of model 29, models 31-32, and model 34, already introduced in section 5 . These are kinematically stable models $\left(R o_{\ell}<0.1\right)$ with mixed mechanical boundary conditions and aspect ratios varying from $\chi=0.5$ to $\chi=0.63$. Models with the same parameter values and boundary conditions but an aspect ratio lower than 0.5 do not exhibit any dynamo action. We therefore considered in addition model 4 with rigid boundary conditions as an example of a dynamo model at lower Rossby number.

Figure 7 shows the growth rates of the fundamental mode and of the first two dipolar overtones versus the local Rossby number for these five models. The fundamental modes have on time average approximately zero growth rate, as it is expected for saturated dynamos, all overtones are diffusive. While there is typically a large gap between the fundamental mode and the first overtone for models at low $R o_{\ell}$, both growth rates are much closer if the Rossby number increases. Quadrupolar modes were omitted in Fig. 7 because the field realized in the DNS is of purely dipolar symmetry for the five examples considered. For completeness, the eigenvalues of the first dipolar and quadrupolar modes are listed in Table 2. We emphasize again that the change in the spectra visible in Fig. 7 is not correlated to an increase of $R m$. The magnetic Reynolds number is highest for model $4(R m=86)$ and does not change significantly for the following models at larger $R o_{\ell}$.

At low Rossby numbers, the fundamental mode is well separated from the following modes (or overtones) which are strongly damped (see Fig. 7). We refer to this characteristic as the 'single-mode' property. As the Rossby number is increased, the average growth rates 


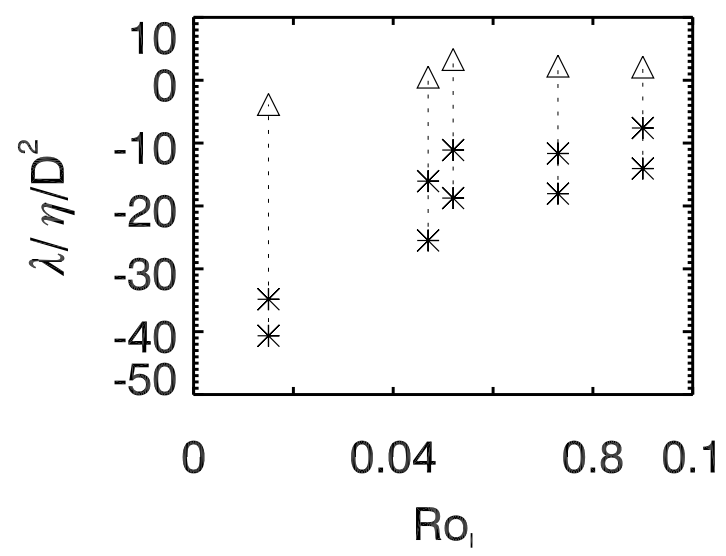

Fig. 7.- Growth rates of the fundamental mode (triangle) and of the first two dipolar overtones (stars) for five models at different Rossby number; in the order of increasing Rossby number, these models are: model 4, model 29, model 31, model 32, and model 34 .

Table 2. Eigenvalues for the first modes with dipolar $\left(\sigma^{a}\right)$ and quadrupolar $\left(\sigma^{s}\right)$ symmetry for the five models considered in Fig. 7 in units of $\eta / L^{2}$.

\begin{tabular}{crrrrr}
\hline \hline Model & \multicolumn{1}{c}{$\sigma_{0}^{a}$} & \multicolumn{1}{c}{$\sigma_{1}^{a}$} & \multicolumn{1}{c}{$\sigma_{2}^{a}$} & \multicolumn{1}{c}{$\sigma_{0}^{s}$} & \multicolumn{1}{c}{$\sigma_{1}^{s}$} \\
\hline \multirow{2}{*}{4} & $(-3.87,0.00)$ & $(-34.8, \pm 10.3)$ & $(-40.6,0.00)$ & $(-6.30,0.00)$ & $(-28.7, \pm 2.69)$ \\
29 & $(0.50,0.00)$ & $(-16.1,0.00)$ & $(-25.5, \pm 4.21)$ & $(-18.3,0.00)$ & $(-25.5, \pm 6.19)$ \\
31 & $(3.30,0.00)$ & $(-11.1,0.00)$ & $(-18.8, \pm 2.75)$ & $(-10.3, \pm 1.70)$ & $(-20.0, \pm 4.88)$ \\
32 & $(2.25,0.00)$ & $(-11.7, \pm 2.51)$ & $(-18.1,0.00)$ & $(-10.0, \pm 2.50)$ & $(-17.5,0.00)$ \\
34 & $(2.10,0.00)$ & $(-7.60,0.00)$ & $(-14.1,0.00)$ & $(-9.35,0.00)$ & $(-10.5,0.00)$ \\
\hline
\end{tabular}



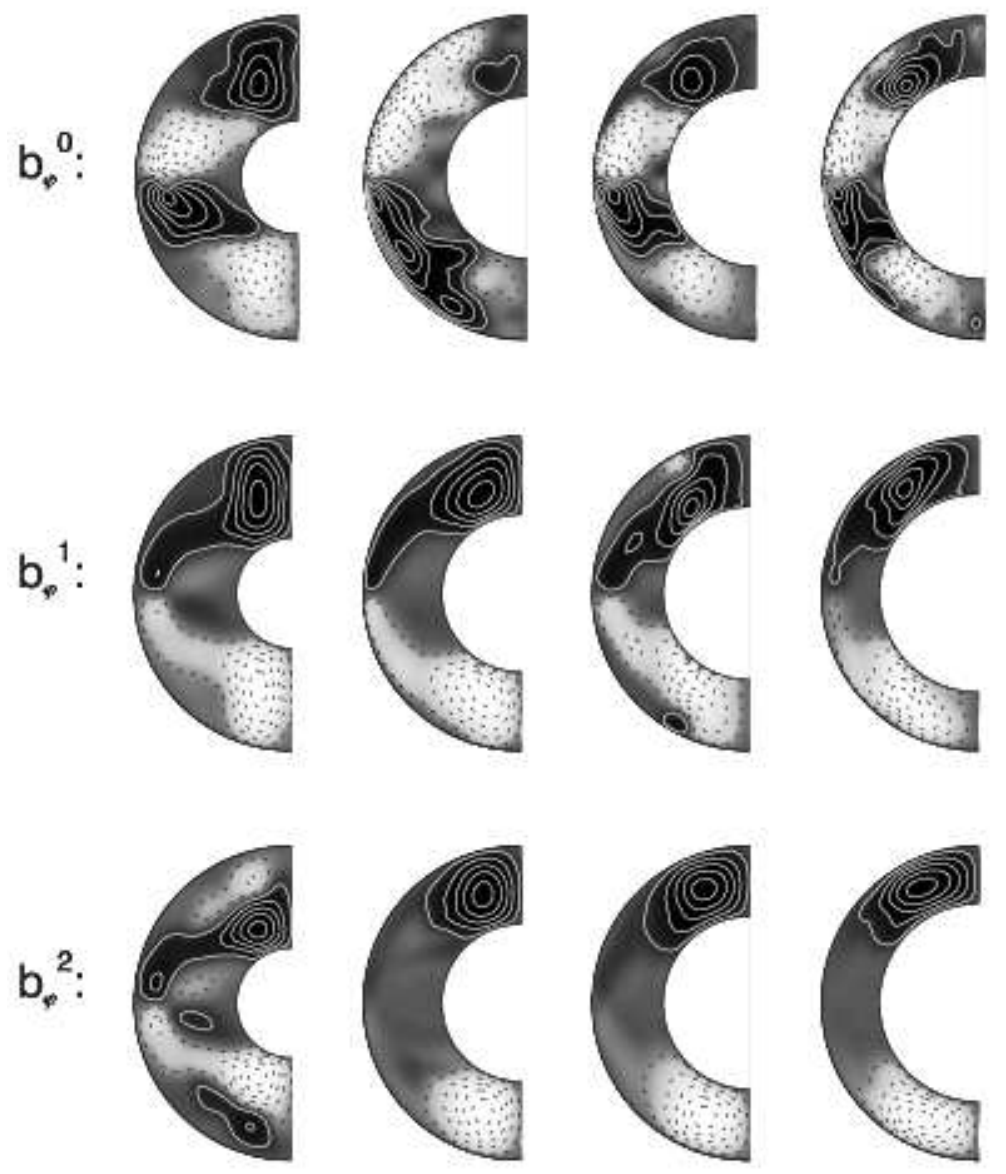

Fig. 8. - Contour plots of the $\varphi$-components of the fundamental mode, $b_{\varphi}^{0}$, and of the first dipolar overtones, $b_{\varphi}^{1}, b_{\varphi}^{2}$, for model 4 , model 29, model 31, and model 34 (in columns from left to right). The first overtone of model 4 (first column) and $b_{\varphi}^{2}$ of model 29 and model 31 are complex and only their real part is shown. The aspect ratio and the local Rossby number increase from model 4 to model 34. Each contour plot is normalized by its maximum absolute value and the gray-scale coding varies from -1 (white) to +1 black. The contour lines correspond to $\pm 0.1, \pm 0.3, \pm 0.5, \pm 0.7$, and \pm 0.9 . 
converge to zero, in other words, the eigenvalues of the overtones approach that of the leading eigenmode. This appears to result from the fact that the structure of the fundamental mode becomes more similar to the following overtones, too. Differences between the first eigenmodes are most visible in their $\varphi$-components. Figure 8 shows contour plots of the fundamental mode and the first two dipolar eigenmodes for model 4, model 29, model 31, and model 34. The axisymmetric azimuthal field of dynamo models at low Rossby number is dominated by two flux bundles of inverse polarity close to the equatorial plane near the outer shell boundary (see also Olson et al. 1999). These flux patches are visible in the $\varphi$ components of the fundamental modes, $b_{\varphi}^{0}$, for all four models. However, as the aspect ratio and the Rossby number increase, the axisymmetric flux concentration at low latitudes becomes less pronounced. The fundamental mode for model 34 resembles in this respect its following overtones and it is probably this adjustment in the eigenmodes which causes the convergence of the eigenvalues for dynamo models at $R o_{\ell} \approx 0.1$.

A systematic change in the field topology of the mean toroidal field with increasing Rossby number can be caused either by a change in the mean flow or by changes in the mean-field coefficients. The first possibility can be ruled out for dipolar models in the low Rossby number regime. For the sequence of models displayed in Figs. 7 and 8, the mean flow does not change significantly. Its influence on the dynamo mechanism is relatively weak and not of primary importance (see also Olson et al. 1999; Schrinner et al. 2007). However, the mean-field coefficients, which are mainly responsible for the generation of the azimuthal field, indeed seem to vary in a systematic manner. Figure 9 displays the dominant dynamo coefficients acting on the toroidal field for model 4, model 29, model 31 and model 34. These are $-\gamma_{r}$ and $-\gamma_{\theta}$ describing an advection of the mean azimuthal field in radial and latitudinal direction, and $-\alpha_{r r}$ and $-\alpha_{\theta \theta}$ leading to the generation of toroidal from poloidal field. We normalized all dynamo coefficients by the maximum modulus of $\alpha_{\varphi \varphi}$ for each model. This third diagonal component of the $\alpha$-tensor is of crucial importance for the generation of poloidal from toroidal field and remains almost unchanged for models at different $R o_{\ell}$.

The $\gamma$-effect advects the mean azimuthal field towards the equatorial plane and the outer shell boundary at low latitudes, and in the opposite direction at higher latitudes and in deeper layers (see also Schrinner et al. 2007). It is directly related to the columnar convection in a spherical shell. A column of fluid elements transported in an upwelling towards the outer spherical boundary has to shorten. Because of mass conservation, this causes a converging flow towards the equatorial plane. Vice versa, the rotational constraint leads to a diverging flow in downwellings in deeper layers and at higher latitudes (Olson et al. 1999). For models approaching $R o_{\ell} \approx 0.1$, the rotational constraint is relaxed and hence the $\gamma$-effect is diminished (see also Fig. 10). In particular, the advective velocity towards the equatorial plane visualized by the outer contours of $-\gamma_{\theta}$ in Fig. 9 is less prominent for 

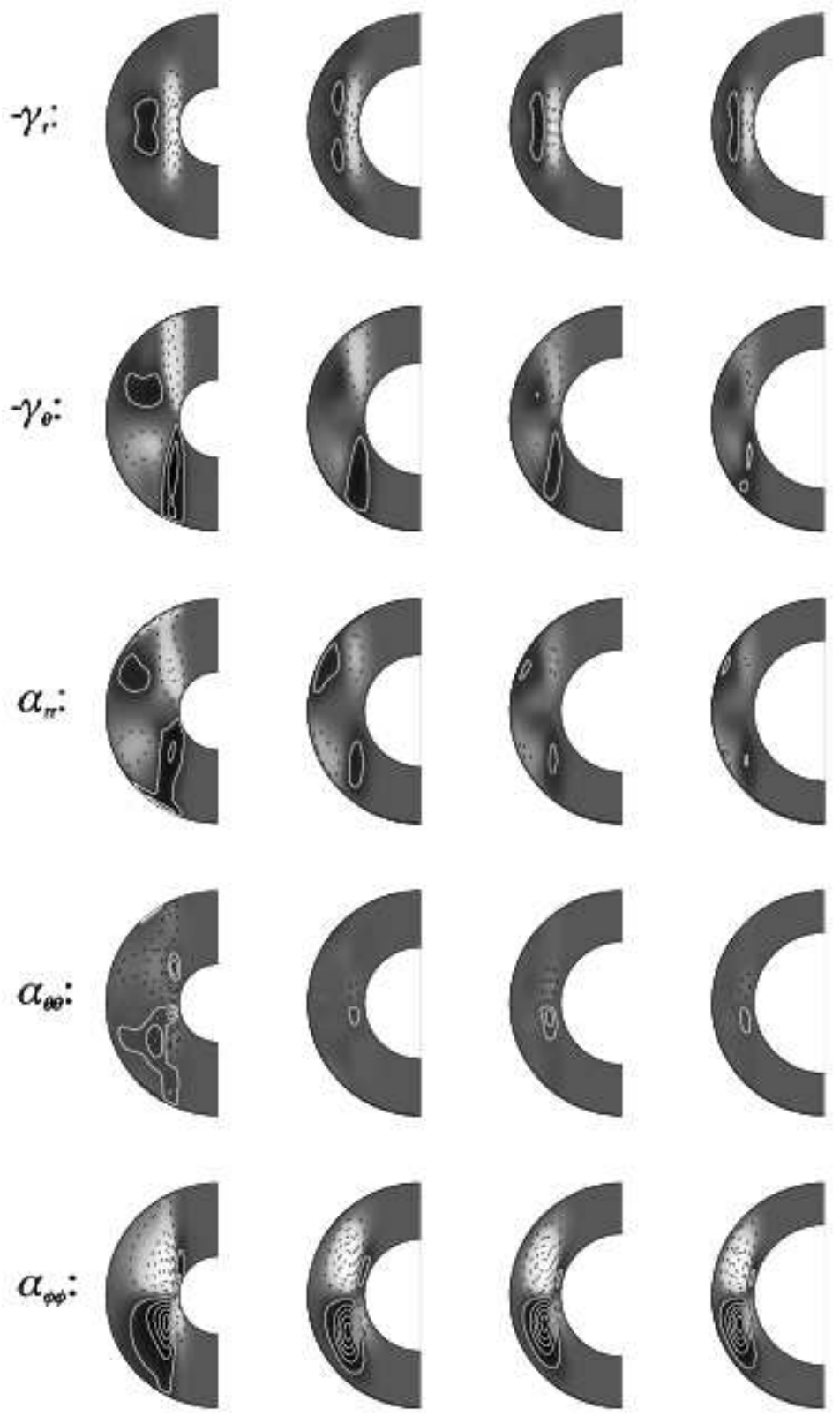

Fig. 9.- Dynamo coefficients for model 4, model 29, model 31 and model 34 (in columns). The mean azimuthal field is advected in radial and latitudinal direction by $-\gamma_{r}$ and $-\gamma_{\theta}$, and generated from poloidal field by $-\alpha_{r r}$ and $-\alpha_{\theta \theta}$. For each model, the dynamo coefficients were normalized by the maximum modulus of $\alpha_{\varphi \varphi}$. White stands for negative and black for positive values. The contour lines are $\pm 0.075, \pm 0.05$, and \pm 0.025 for $\alpha_{\theta \theta}$ and as in Fig. 8 for all other coefficients. 
model 34 than for model 4 . This is consistent with the changes in the topology of $b_{\varphi}^{0}$ for these models.

The significance of the $\gamma$-effect in dipolar dynamo models is demonstrated by a meanfield calculation, in which $\gamma$ was arbitrarily suppressed. Figure 11 shows the azimuthally and time-averaged magnetic field for model 34 obtained from DNS (first row) and the leading eigenmode of a corresponding mean-field calculation based on all mean-field coefficients determined (second row). Both are in good agreement. If the dynamo-coefficients related to the $\gamma$-effect are set to zero in a numerical experiment, the result changes substantially. The eigenvalue spectrum is flat and there are two complex, growing eigenmodes of either symmetry, i.e. the model is no longer kinematically stable. The resulting first real, dipolar eigenmode shown in the third row of Fig. 11 varies on fairly small length scales. Moreover, the mean azimuthal flux concentrations at low latitudes near the outer boundary characteristic for dipolar dynamo models disappeared.

Similar to the $\gamma$-components, $\alpha_{r r}$ decreases considerably with increasing Rossby number, as demonstrated clearly by Fig. 10. It is the dominating coefficient responsible for the toroidal field generation by an $\alpha$-effect. At low Rossby numbers, a strong $\gamma$-effect leads to the distinctive azimuthal field configuration with two flux bundles of inverse polarity close to the equatorial plane. The also increased $\alpha_{r r}$-component in this dynamo regime sustains it against the efficient diffusion due to strong gradients necessarily related to this field topology. The $\alpha_{\theta \theta}$-component, on the other hand, is on average much smaller than $\alpha_{r r}$ and remains at low level independent of the Rossby number. 

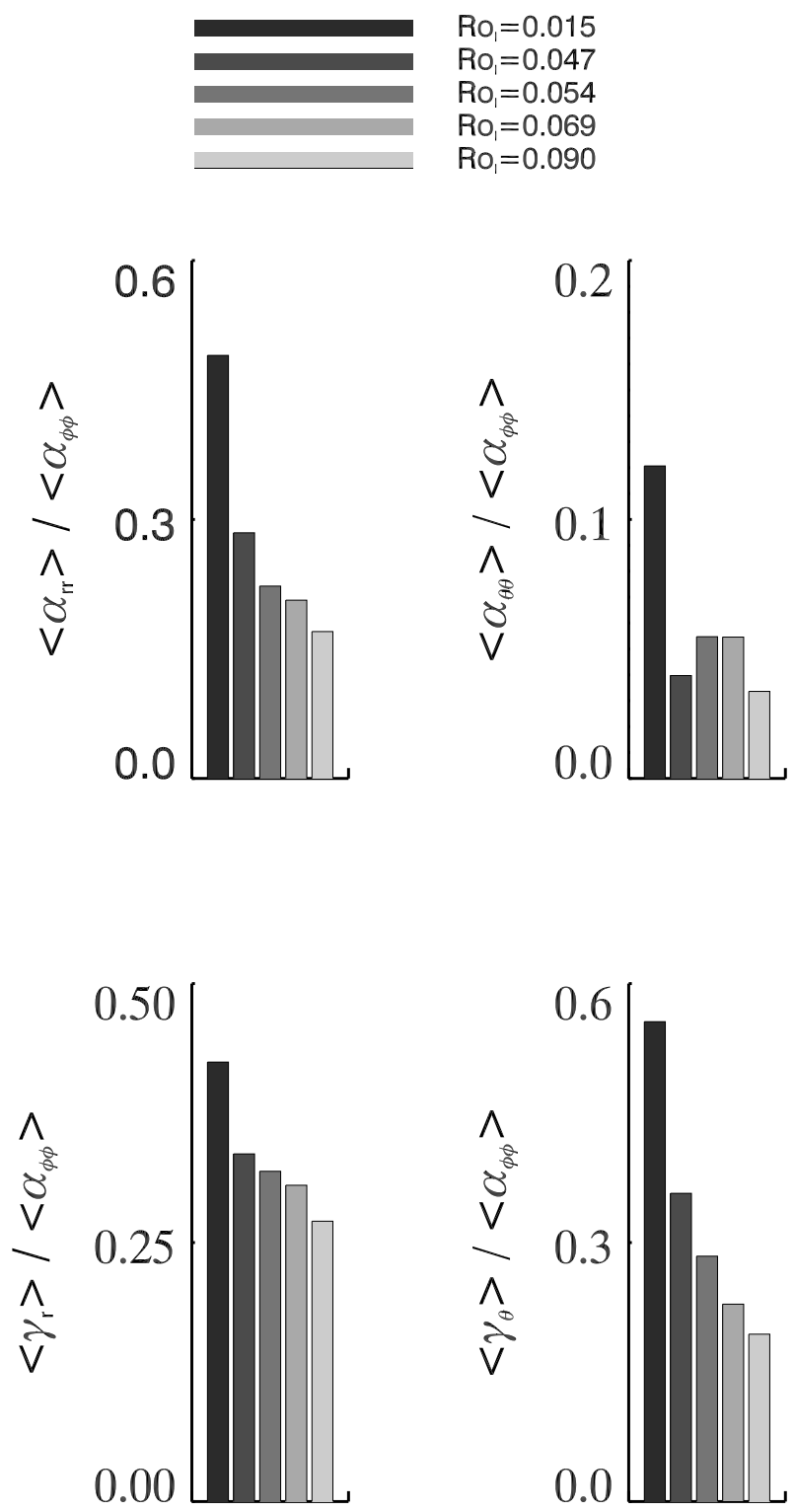

Fig. 10. - The time and spatially averaged values of $\alpha_{r r}, \alpha_{\theta \theta}, \gamma_{r}$, and $\gamma_{\theta}$ normalized by the mean value of $\alpha_{\phi \phi}$ for model 4, model 29, model 31, model 32, and model 34. 


\section{$\mathrm{B}_{\mathrm{r}}$}
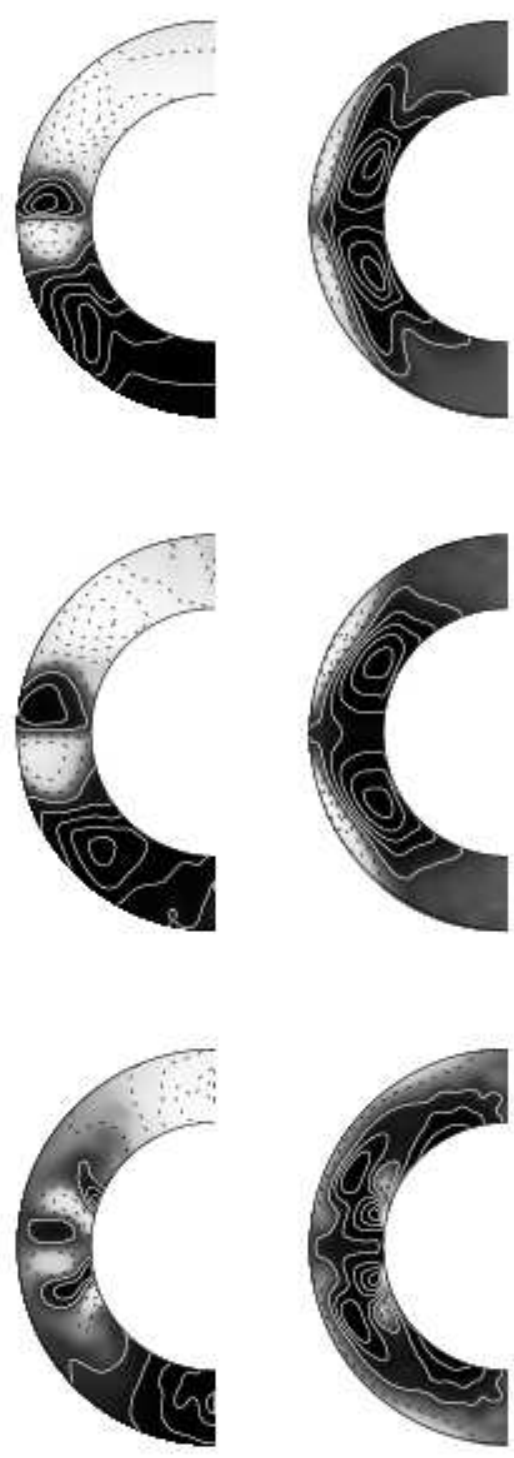

$\mathrm{B}_{\vartheta}$
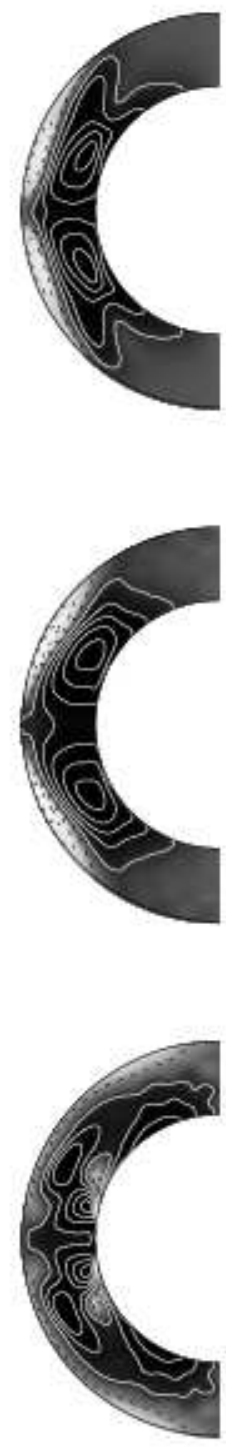
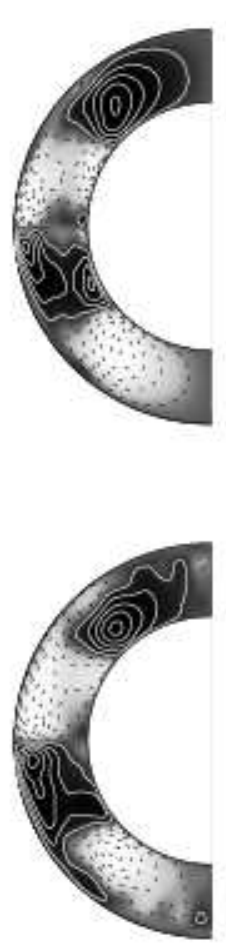

$\mathrm{B}_{\varphi}$

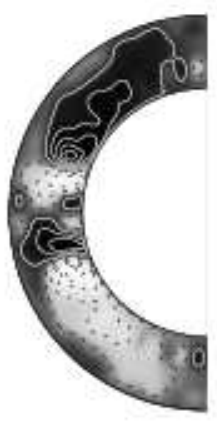

Fig. 11.- First row: Azimuthally and time-averaged magnetic field for model 34 obtained by direct numerical simulations. Second row: Leading eigenmode derived from (77) with the complete dynamo operator for model 34 as defined in (8). Third row: Eigenmode derived from (7) with a dynamo operator for model 34 in which the $\gamma$-effect was artificially suppressed. Each component is normalized by its maximum modulus and the gray-scale coding varies from -1 (white) to +1 (black). The contour lines correspond to $\pm 0.1, \pm 0.3, \pm 0.5, \pm 0.7$, and \pm 0.9 . 


\subsection{Reason for the bistability and dynamo waves}

The existence of a dipolar and a multipolar dynamo regime with a sharp transition between both at $R o_{\ell} \approx 0.1$ does not depend on the choice of the mechanical boundary conditions. The transition here is therefore not controlled by the thickness of the Ekman boundary layers (as opposed to King et al. 2009). However, most of the models with stressfree conditions at both boundaries and $R_{\ell}<0.1$ exhibit a second, non-dipolar magnetic-field branch. Stress-free mechanical boundary conditions allow for the development of a strong, axisymmetric azimuthal flow, if the magnetic field is initially weak. In the presence of stress-free boundary conditions, the zonal geostrophic flow can only saturate owing to bulk viscosity and magnetic forces. When no-slip boundaries are present, the viscous braking of the geostrophic flow occurs mainly in boundary layers, an effect much stronger (by a factor

$E^{-1 / 2}$ ) than bulk viscous effects (e.g. Morin \& Dormy 2006). As a result, for stress-free conditions at the inner and the outer shell boundaries, even a very weak inertial forcing (Reynolds stresses) can yield a very significant geostrophic flow if the magnetic field is weak. The zonal flow pattern is then highly geostrophic, i.e. $\bar{V}_{\varphi}$ is constant on cylinders parallel to the rotation axis. Conversely, for the dipolar branch, the development of a zonal flow is partly inhibited by Maxwell stresses, leading to substantial deviations from geostrophy (as also observed by Busse \& Simitev 2009). The difference between both flow profiles is visible in Fig. 12 for model $45 \mathrm{~m}$ and model $45 \mathrm{~d}$. Furthermore, the variation in the mean zonal flow causes differences in the $\Omega$-effect, $r \overline{\boldsymbol{B}}_{r} \partial\left(r^{-1} \overline{\boldsymbol{V}}_{\phi}\right) / \partial r+r^{-1} \sin \theta \overline{\boldsymbol{B}}_{\theta} \partial\left(\sin \theta^{-1} \overline{\boldsymbol{V}}_{\phi}\right) / \partial \theta$, and thus in the dynamo mechanism for the toroidal field of both branches. For model $45 \mathrm{~m}$, the $\Omega$-effect correlates nicely with the mean azimuthal field (upper panel of Fig. 12), and therefore, the model may be characterized as an $\alpha \Omega$-dynamo. However, for model $45 \mathrm{~d}$, the mean azimuthal field is for the most part not the result of the $\Omega$-effect. In particular the flux portions at larger radii and close to the equatorial plane are, if at all, anticorrelated to it.

The difference in the dynamo mechanism for both branches explains why the Rossby number criterion for the dipolarity of the magnetic field applies only to the dipolar branch. The Rossby number criterion as formulated in this work compares the convective length scale with the Rossby radius. If the magnetic field is not solely a result of columnar convection but its generation mechanism also involves a large-scale zonal flow, the Rossby number criterion becomes meaningless. Other examples for which it does not apply, presumably for the same reason, can be found in Hori et al. (2010) and Landeau \& Aubert (2011). Similar to models at high $R o_{\ell}$, model $45 \mathrm{~m}$ lacks the particular azimuthal field configuration typical for dipolar or 'single-mode' dynamos. The magnetic field is governed on this branch by several modes and the relative dipole field strength drops to $f_{\text {dip }}=0.25$. The model is kinematically unstable and the magnetic field exhibits quasi-periodic time variations (see Fig. 51). 
Dynamo models of this stress-free multipolar branch and those with $R o_{\ell}>0.1$ are, for different reasons, not dominated by a dipolar mode. The dominance of only one, real dipolar eigenmode associated with the columnar flow is broken and in general complex overtones play an essential role in the dynamics of the magnetic field. If the magnetic Reynolds number is sufficiently low, the magnetic field evolves in the form of coherent dynamo waves. However, with increasing distance of the models from the dynamo threshold, the temporal coherence is lost presumably due to the enlarged number of relevant modes. A particular dynamo mechanism, on the other hand, is not the primary reason for oscillatory dynamos. Models $35-38\left(R_{\ell}>0.1\right)$ exhibit fairly coherent dynamo waves, but they are not of a $\alpha \Omega$-type (see Schrinner et al. 2011). For these examples, oscillatory dynamos are found because the rotational constraint is relaxed, i.e. $R o_{\ell}>0.1$, and nevertheless $R m$ remains moderate $(R m \lesssim 200)$. This twofold condition can be fulfilled for models with a thin convection zone, for example, and governs the transition from steady to oscillatory dynamos already highlighted by Goudard \& Dormy (2008).

Christensen \& Aubert (2006) pointed out that the cube of the magnetic field strength for models with rigid boundaries is proportional to the measured buoyancy flux. The Lorentz number and the flux-based Rayleigh number, $R a_{Q}=R a(N u-1) E^{2} / P r$, can then be related trough

$$
\frac{L o}{f_{\mathrm{ohm}}^{1 / 2}} \propto R a_{Q}^{1 / 3},
$$

where $f_{\mathrm{ohm}}$ is the ratio of ohmic to total dissipation. The coefficient of proportionality was determined, still in the case of rigid boundaries, to be 0.79 for dipolar models and 0.48 for multipolar models (Christensen 2010). We found, see Fig 13, that the magnetic field strength for our limited sample of stress-free models is consistent with this relation, with similar prefactors.

As noted already by Busse \& Simitev (2011), there is almost no difference in the Nusselt number, and thus in the flux-based Rayleigh number, for pairs of dipolar and multipolar models in the bistable regime (see Table 31). However, $f_{\text {ohm }}$ is always smaller for the multipolar branch, i.e. the $\alpha \Omega$-mechanism is less efficient than the $\alpha^{2}$-mechanism related to columnar convection. This deficiency together with the somewhat lower prefactor relates to for the lower field strength found for these models.

\subsection{Bifurcations between dynamo branches}

It is interesting to ponder on the transitions between the dipolar and multipolar branch

for stress-free models when one single control parameter is varied. The two branches are 


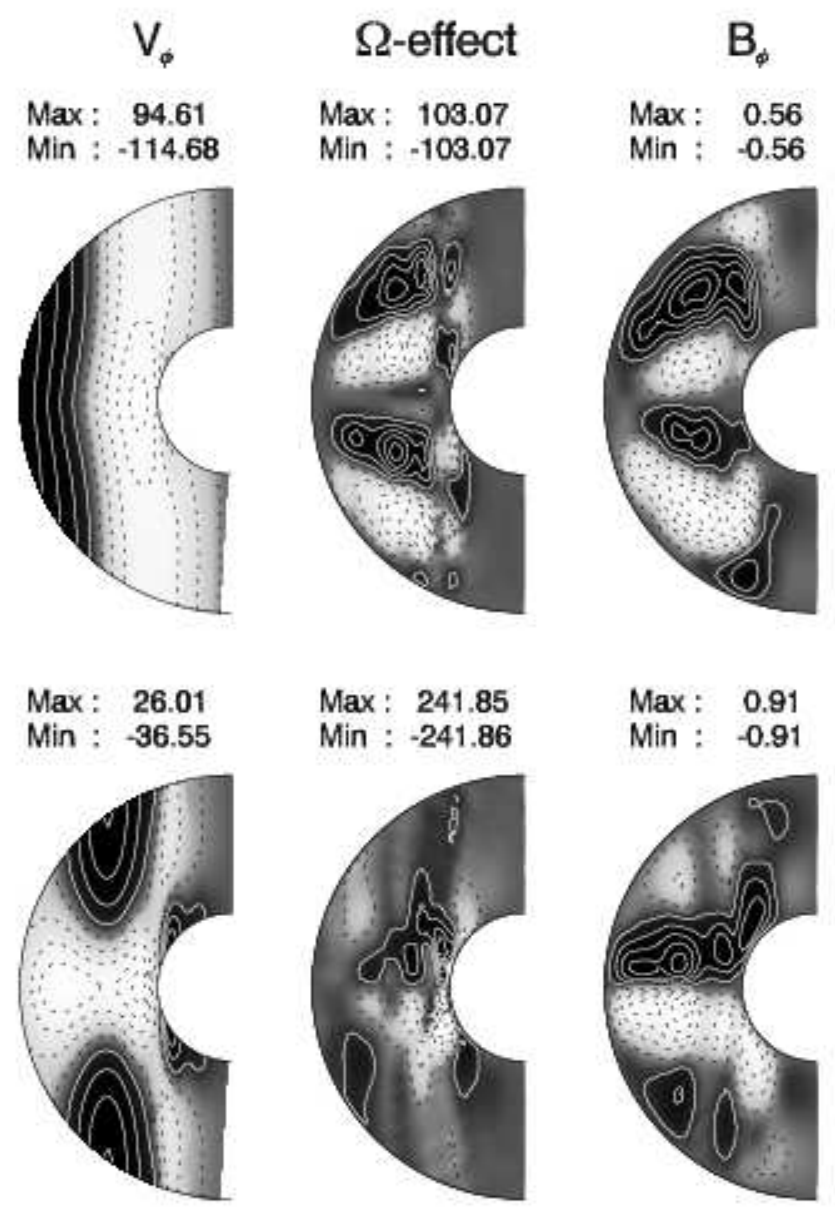

Fig. 12.- The axisymmetric azimuthal flow, the $\Omega$-effect expressed as $r \overline{\boldsymbol{B}}_{r} \partial\left(r^{-1} \overline{\boldsymbol{V}}_{\phi}\right) / \partial r+$ $r^{-1} \sin \theta \overline{\boldsymbol{B}}_{\theta} \partial\left(\sin \theta^{-1} \overline{\boldsymbol{V}}_{\phi}\right) / \partial \theta$, and the axisymmetric azimuthal magnetic field for model $45 \mathrm{~m}$ (upper panel) and model 45d (lower panel). The style of the contour plots is explained in the caption of Fig. 11.

Table 3. Nusselt number and ratio of ohmic to total dissipation, $f_{\mathrm{ohm}}$, for bistable models with stress-free boundary conditions

\begin{tabular}{|c|c|c|c|c|c|c|c|c|c|c|c|c|c|c|c|c|}
\hline \multicolumn{17}{|c|}{$\mathrm{Nu}$ and $f_{\mathrm{ohm}}$ for models with stress-free boundary conditions } \\
\hline Model & $43 \mathrm{~m}$ & $43 \mathrm{~d}$ & $45 \mathrm{~m}$ & $45 \mathrm{~d}$ & $46 \mathrm{~m}$ & $46 \mathrm{~d}$ & $47 \mathrm{~m}$ & $47 \mathrm{~d}$ & $48 \mathrm{~m}$ & $48 \mathrm{~d}$ & $49 \mathrm{~m}$ & $49 \mathrm{~d}$ & $51 \mathrm{~m}$ & $51 \mathrm{~d}$ & $52 \mathrm{~m}$ & $52 \mathrm{~d}$ \\
\hline $\mathrm{Nu}$ & 2.0 & 1.9 & 2.2 & 2.4 & 3.0 & 3.0 & 3.0 & 3.1 & 2.9 & 2.9 & 2.3 & 2.5 & 1.3 & 1.4 & 4.3 & 4.2 \\
\hline$f_{\mathrm{ohm}}$ & 0.29 & 0.48 & 0.28 & 0.43 & 0.32 & 0.56 & 0.30 & 0.56 & 0.31 & 0.42 & 0.25 & 0.47 & 0.48 & 0.58 & 0.38 & 0.59 \\
\hline
\end{tabular}




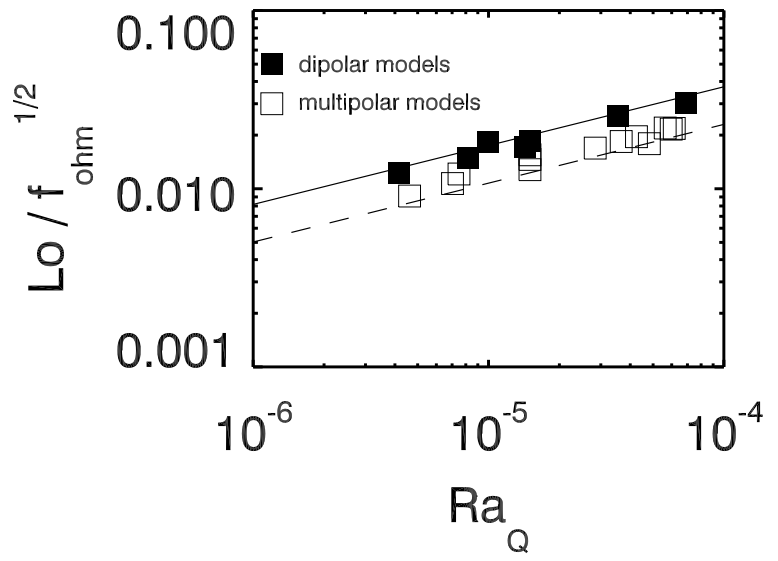

Fig. 13. - Lorentz number corrected by the dissipation factor versus the flux based Rayleigh number. The straight lines represent the scaling for dipolar and multipolar models with rigid boundaries (Christensen 2010). Our models with stress-free boundaries (squares stand for dipolar and diamonds for multipolar models) follow a similar scaling.

illustrated in Fig. 14 for a fixed Ekman number of $E=10^{-4}$ and magnetic Prandtl number of $P m=1$. For both branches, the local Rossby number increases with increasing Rayleigh numbers (see Table 1). If the Rayleigh number is increased from $R a=1110$ (model 52d) on the dipolar branch to $R a=1200$, the relative dipole field strength collapses (the local Rossby number crosses the $R o_{\ell} \sim 0.1$ boundary). The multipolar field configuration then appears to be the only stable solution (circle on the figure) and a hysteretic behavior is observed if the Rayleigh number is decreased from this state (i.e. model $52 \mathrm{~m}$ is then obtained).

Interestingly, the transition between both branches is not always as abrupt as in Fig. 14. Instead, the two branches can also merge more continuously if the zonal geostrophic flow on the multipolar branch becomes too weak. This is best demonstrated by varying the magnetic Prandtl number keeping all other control parameters fixed. Figure 15 presents the axisymmetric toroidal kinetic energy as a function of the magnetic Prandtl number at fixed Rayleigh number $(R a=750)$ and Ekman number $\left(E=10^{-4}\right)$. Both branches are represented (the multipolar solution corresponding to the larger values). If the magnetic Prandtl number is increased the saturated value of the geostrophic flow on the multipolar branch decreases. For $P m=6$ (circle on the figure) the multipolar solution is only metastable (see Morin \& Dormy 2009). It can be observed for a short period of time (enough to assess its amplitude) but then switches to the dipolar solution. For this value of the control parameter $(\mathrm{Pm})$, only the dipolar solution could be produced. The saturated amplitude of 
the geostrophic flow is here too small to prevent the growth of the dipolar solution. This behavior is typical for simulations at large $P m$, although the value at which the multipolar branch is lost depends on the other parameters, too.

Finally, besides the transition between both branches, it is worth pondering on how these dynamo solutions bifurcate from the purely hydrodynamic solution. We have not performed a detailed study of this problem, but we observed in Fig. 15 that for Pm= 0.5 , both solutions exist, while the hydrodynamic solution is stable (i.e. a small magnetic perturbation decays, and a finite amplitude perturbation is needed to obtain any of the dynamo solutions). The subcritical bifurcation of the dipolar mode at low Pm was already described in Morin \& Dormy (2009) with no-slip boundary conditions. It is interesting that the system exhibits here a triple stability (two dynamo solutions and a purely hydrodynamic mode). Obviously the dynamo bifurcation with stress-free boundary conditions deserves further studies. 


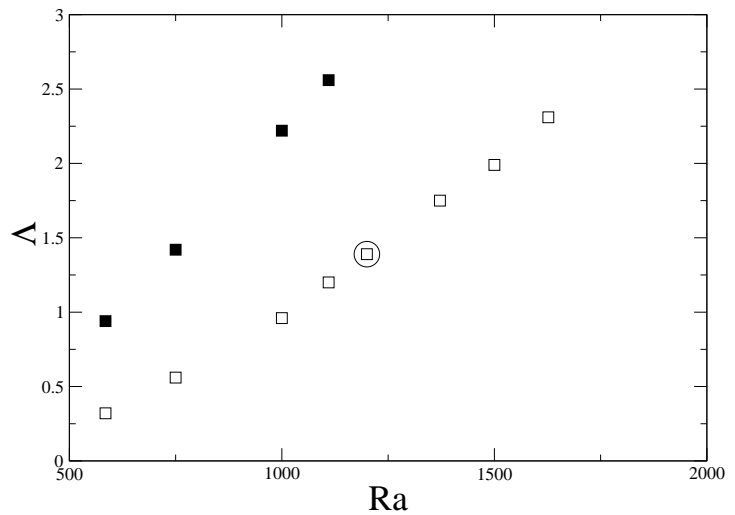

Fig. 14.- Evolution of the magnetic field strength, measured by the Elsasser number, for both branches as the Rayleigh number is varied at fixed Ekman and magnetic Prandtl numbers $\left(E=10^{-4}\right.$ and $\left.P m=1\right)$.

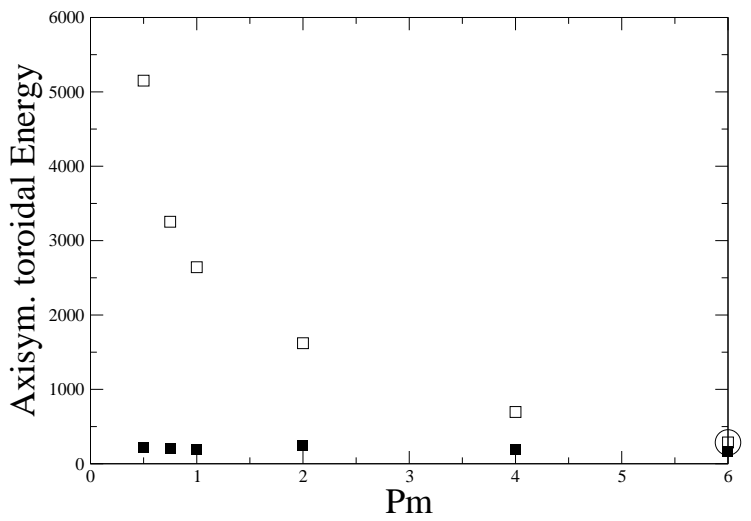

Fig. 15.- Axisymmetric toroidal energy density of the flow as a function of the magnetic Prandtl number at fixed Rayleigh number $(R a=750)$ and Ekman number $\left(E=10^{-4}\right)$ defined as one half of the average over the fluid volume of the non-dimensional toroidal velocity squared. 


\subsection{Dynamo models versus stellar and planetary dynamos}

It is not known to which extent simplified dynamo models indeed reflect physical processes going on in stellar or planetary dynamos. All simulations to date are performed in a wrong parameter regime; they are therefore not directly comparable with observations. However, scaling laws derived from numerical dynamo simulations could help to test their reliability. Results obtained from dynamo models may be compared with the strength, the geometry and the time dependence of stellar and planetary magnetic fields.

The relation between the field strength and the flux-based Rayleigh number (9) proposed by Christensen \& Aubert (2006) is consistent with the field-strength for a class of fast rotating stars and some of the planets (Christensen et al. 2009). However, without further assumptions, it is neither applicable to slowly rotating stars, e.g. the Sun, nor

to Mercury, Saturn, Uranus and Neptune (Christensen 2010). For theses examples, the field strength falls below the predicted value. Moreover, Morin et al. (2010) observed M dwarfs of very similar mass (i.e. energy flux) and rotation rate, but with dynamo generated magnetic fields which differ in their field strength and their field topologies. Morin et al. (2011) argued that a different force balance could be responsible for the observed bistability, similar to the strong-field branch scenario introduced in the context of the geodynamo (Roberts, P. H. \& Soward, A. M. 1978; Roberts 1988). In this study, we point out that differences in the dynamo mechanism could likewise lead to two different magnetic field branches. The shearing of poloidal field lines ( $\Omega$-effect) due to a strong geostrophic zonal flow may play an important role for the field generation in multipolar, but not in dipolar models. A dynamo mechanism based on the action of a mean zonal flow leads to models characterized by a more variable and a somewhat weaker magnetic field. The bistability investigated in this study was found for a wide range of parameter values. It could also account for different magnetic fields of stars with similar parameters at a Rossby number close to 0.1 , which has not been observed yet.

The Rossby number criterion for the dipolarity of the magnetic field is consistent with the topology of planetary magnetic fields, except for Uranus and Neptune (see Christensen 2010). Furthermore, it gets some support from observations of stellar magnetic fields (Morin et al. 2008). In particular, a decrease of the size of the convection zone may lead to higher local Rossby numbers and thus to an abrupt change in the field topology, as observed for early $\mathrm{M}$ dwarfs (see Fig. 2 and Morin et al. 2008; Donati et al. 2008; Reiners \& Basri 2009). Likewise, the multipolar magnetic fields of Uranus and Neptune could be compatible with the Rossby number rule, if convection in these planets takes place in a thin convection zone, as suggested by Stanley \& Bloxham (2004, 2006).

The regime boundary at $R o_{l} \approx 0.1$ separates models with a stable dipole field from 
models exhibiting dipole reversals. Why are models of the dipolar regime non-reversing? The fact that modes, other than the fundamental mode, are on average damped does not prevent reversals, which may be triggered by the coupling with a weakly damped competing dynamo mode (Pétrélis et al. 2009). But the 'single mode property' of these models reported above excludes that different modes become critical and explains why a reversal mechanism based on the coupling of competing modes has not been identified in these models. It has been proposed that the value of $R o_{\ell}$ for the Earth's core may be about 0.1 and thus in the vicinity of the regime boundary, which could also provide a mechanism for polarity reversals (Olson \& Christensen 2006).

Whether stellar and planetary magnetic fields are dipolar for the same reason as the models could perhaps be assessed studying their time dependence. The single mode property should lead to different time scales for the variation of the dipolar and the nondipolar field (Schrinner et al. 2011), as it has been reported for the geomagnetic secular variation (e.g. Le Mouël 1984). Tanriverdi \& Tilgner (2011) recently pointed out that single mode dynamos may be identified by the spectrum of temporal fluctuations of the magnetic energy. If the velocity spectrum is characterized by white noise and the evolution of the magnetic field is dominated by only one single dynamo mode, a $\omega^{-2}$ dependence for the spectrum of the magnetic field was predicted. This might be verifiable with magnetic field data. As discussed above, reversals of the Earth's magnetic field clearly indicate that the geodynamo cannot always match the single mode property.

We found coherent magnetic cycles in our non-dipolar dynamo models only if the mag-

netic Reynolds number is sufficiently low. Similarly, Brown et al. (2011) reports oscillations of the magnetic field in an anelastic simulation at fairly low magnetic Reynolds number, $R m=136$. If $R m$ is increased in our simulations, the temporal coherence is lost. Moreover, the dynamo waves in our models migrate from low latitudes towards the poles (see also Schrinner et al. 2011), as opposed to solar sunspot regions. Given that estimates for the magnetic Reynolds number range from $10^{6}$ in the photosphere to $10^{10}$ at the base of the convection zone (Ossendrijver 2003), it remains unclear how the temporal coherence visible in the 22-year solar cycle can persist in such a highly turbulent environment (Jones et al. 2010).

\section{Conclusions}

Convection in a rapidly rotating spherical shell is organized in quasi-geostrophic columns parallel to the rotation axis. It gives rise to highly efficient but also very selective dynamo action: only one, real, dipolar eigenmode of the magnetic field is sustained. This single-mode 
property accounts for the dipole dominance and the stability of the dipole field in models of this regime. Consequently, the dipolarity of our models collapses, if the dominance of the fundamental mode is broken and, in general, complex overtones dominate the evolution of the magnetic field. This may happen if convection is less constrained by rapid rotation, or, if the magnetic field is not solely a result of columnar convection, but a mean zonal flow strongly influences the dynamo mechanism. Whether or not differences in the topology and the time variability of planetary and stellar magnetic fields may be explained by the dichotomy between these two dynamo regimes certainly needs to be further explored.

We acknowledge discussions with J.F. Donati and J. Morin. MS is grateful for financial support from the DFG fellowship SCHR 1299/1-1. LP and ED acknowledge financial support from "Programme National de Physique Stellaire" (PNPS) of CNRS/INSU, France. Computations were performed at CINES and CEMAG computing centers.

\section{A. Appendix}

The definition of the local Rossby number given here relies on a length scale derived from the mean harmonic degree of only the poloidal velocity field. This intends to filter out the contribution of the mean toroidal flow, which is not negligible in models with stress-free mechanical boundary conditions. The local Rossby number introduced by Christensen \& Aubert (2006) based on a length scale derived from the mean harmonic degree of the total flow does not allow us to distinguish dipolar models from multipolar models equally well (Fig. [16, left panel).

In this study we argued that the typical length scale of convection relative to the Rossby radius strongly influences the topology of the magnetic field in our models. The definition of a local Rossby number based on a velocity field, in which only the axisymmetric toroidal contribution is canceled out is maybe more appropriate to test this argument than the definition used throughout in the text. The right panel of Fig. 16] shows that a local Rossby

number defined in this way seems to serve equally well to distinguish dipolar from multipolar models. 

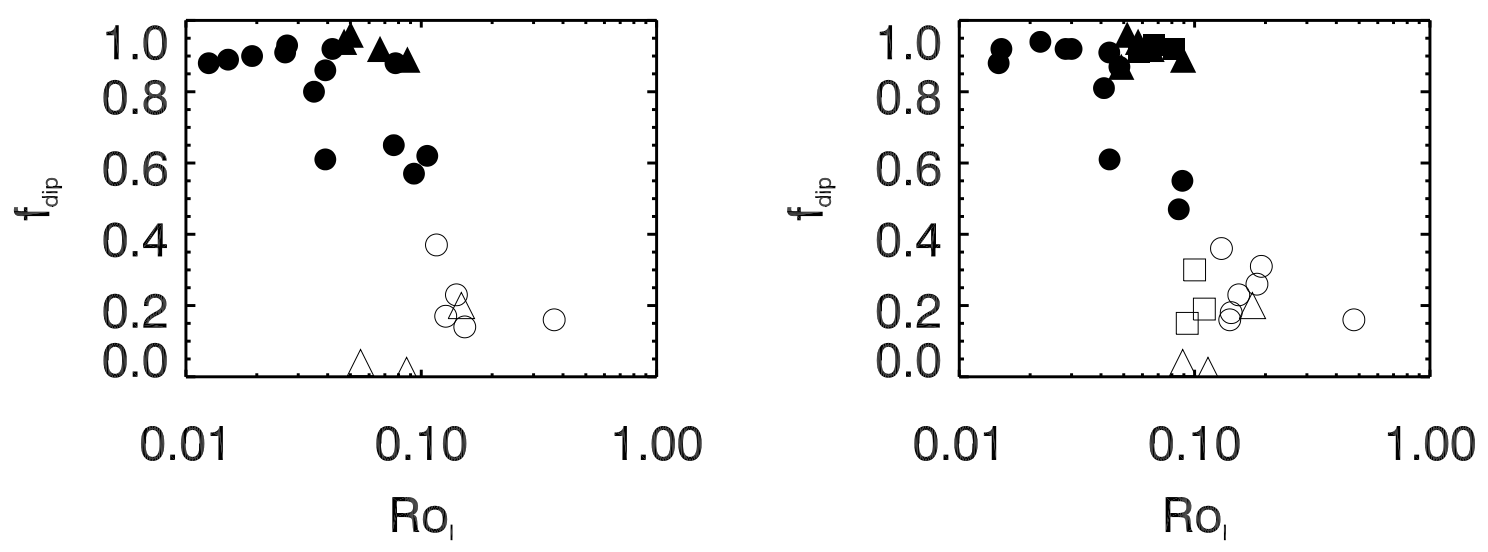

Fig. 16. - Relative dipole field strength versus local Rossby number. On the left, the local Rossby number is based on a length scale derived from the mean harmonic degree of the total flow as introduced by Christensen \& Aubert (2006), on the right, it is based on a velocity field and its typical length scale without considering the contribution of the mean toroidal flow component. The meaning of the symbols is the same as in Fig. 1, 


\section{REFERENCES}

Anderson, B. J., et al. 2010, Space Sci. Rev., 152, 307

Brown, B. P., Browning, M. K., Brun, A. S., Miesch, M. S., \& Toomre, J. 2010, ApJ, 711, 424

Brown, B. P., Miesch, M. S., Browning, M. K., Brun, A. S., \& Toomre, J. 2011, ApJ, 731, 69

Busse, F., \& Simitev, R. 2011, Geophysical and Astrophysical Fluid Dynamics, 105, 234

Busse, F. H., \& Simitev, R. D. 2009, ArXiv e-prints

Cattaneo, F., \& Tobias, S. M. 2009, J. Fluid Mech., 621, 205

Christensen, U. R. 2010, Space Sci. Rev., 152, 565

Christensen, U. R., \& Aubert, J. 2006, Geophy. J. Int., 166, 97

Christensen, U. R., Holzwarth, V., \& Reiners, A. 2009, Nature, 457, 167

Christensen, U. R., et al. 2001, Phys. Earth Planet. Inter., 128, 25

Donati, J.-F., \& Landstreet, J. D. 2009, ARA\&A, 47, 333

Donati, J.-F., et al. 2008, MNRAS, 390, 545

Dormy, E., Cardin, P., \& Jault, D. 1998, Earth Planet. Sci. Lett., 160, 15

Glatzmaier, G. A. 1984, Journal of Computational Physics, 55, 461

Goudard, L., \& Dormy, E. 2008, Europhys. Lett., 83, 59001

Hori, K., Wicht, J., \& Christensen, U. R. 2010, Physics of the Earth and Planetary Interiors, 182,85

Hoyng, P. 2009, Phys. Rev. E, 79, 046320

Hulot, G., Finlay, C. C., Constable, C. G., Olsen, N., \& Mandea, M. 2010, Space Sci. Rev., 152,159

Jones, C. A., Thompson, M. J., \& Tobias, S. M. 2010, Space Sci. Rev., 152, 591

Käpylä, P. J., Korpi, M. J., \& Brandenburg, A. 2009, ApJ, 697, 1153 
King, E. M., Stellmach, S., Noir, J., Hansen, U., \& Aurnou, J. M. 2009, Nature, 457, 301

Krause, F., \& Rädler, K. 1980, Mean-field magnetohydrodynamics and dynamo theory (Oxford: Pergamon Press)

Kutzner, C., \& Christensen, U. R. 2002, Physics of the Earth and Planetary Interiors, 131, 29

Landeau, M., \& Aubert, J. 2011, Physics of the Earth and Planetary Interiors, 185, 61

Le Mouël, J. L. 1984, Nature, 311, 734

Moffatt, H. K. 1978, Magnetic field generation in electrically conducting fluids (Cambridge: Cambridge University Press)

Morin, J., Donati, J.-F., Petit, P., Delfosse, X., Forveille, T., \& Jardine, M. M. 2010, MNRAS, 407, 2269

Morin, J., Dormy, E., Schrinner, M., \& Donati, J.-F. 2011, MNRAS, 418, L133

Morin, J., et al. 2008, MNRAS, 390, 567

Morin, V., \& Dormy, E. 2006, Physics of Fluids, 18, 068104

—. 2009, International Journal of Modern Physics B, 23, 5467

Olson, P., \& Christensen, U. R. 2006, Earth and Planetary Science Letters, 250, 561

Olson, P., Christensen, U. R., \& Glatzmaier, G. A. 1999, J. Geophys. Res., 104, 10383

Ossendrijver, M. 2003, A\&A Rev., 11, 287

Parker, E. N. 1955, ApJ, 122, 293

Pétrélis, F., Fauve, S., Dormy, E., \& Valet, J. 2009, Phys. Rev. Lett., 102, 144503

Rädler, K. H. 1969a, Monats. Dt. Akad. Wiss., Berlin, Volume 11, p. 272-279, 11, 272

—. 1969b, Monats. Dt. Akad. Wiss., Berlin, Volume 11, p. 194-201, 11, 194

Rädler, K.-H. 1995, in Reviews in Modern Astronomy, Vol. 8, Rev. Mod. Astron., ed. G. Klare, 295-322

Reiners, A., \& Basri, G. 2009, A\&A, 496, 787

Roberts, P. H. 1988, Geophysical and Astrophysical Fluid Dynamics, 44, 3 
Roberts, P. H. \& Soward, A. M., ed. 1978 (London: Academic Press)

Russell, C. T., \& Dougherty, M. K. 2010, Space Sci. Rev., 152, 251

Schrinner, M. 2011, A\&A, 533, A108+

Schrinner, M., Petitdemange, L., \& Dormy, E. 2011, A\&A, 530, A140

Schrinner, M., Rädler, K.-H., Schmitt, D., Rheinhardt, M., \& Christensen, U. R. 2005, Astron. Nachr., 326, 245

—. 2007, Geophys. Astrophys. Fluid Dyn., 101, 81

Schrinner, M., Schmitt, D., Cameron, R., \& Hoyng, P. 2010a, Geophys. J. Int., 182, 675

Schrinner, M., Schmitt, D., \& Hoyng, P. 2011, Physics of the Earth and Planetary Interiors,

Schrinner, M., Schmitt, D., Jiang, J., \& Hoyng, P. 2010b, A\&A, 519, A80+

Simitev, R. D., \& Busse, F. H. 2009, EPL (Europhysics Letters), 851, 19001

Sreenivasan, B., \& Jones, C. A. 2006, Geophysical Journal International, 164, 467

—. 2011, Journal of Fluid Mechanics, 688, 5

Stanley, S., \& Bloxham, J. 2004, Nature, 428, 151

-. 2006, Icarus, 184, 556

Steenbeck, M., Krause, F., \& Rädler, K. 1966, Zeit. Nat. A, 21, 369

Stellmach, S., \& Hansen, U. 2004, Phys. Rev. E, 70, 056312

Tanriverdi, V., \& Tilgner, A. 2011, New Journal of Physics, 13, 033019

Tilgner, A., \& Brandenburg, A. 2008, MNRAS, 391, 1477

This preprint was prepared with the AAS LATEX macros v5.2. 